\title{
The association between physical activity with incident obesity, coronary heart disease, diabetes and hypertension in adults: a systematic review of longitudinal studies published after 2012
}

\author{
Laura Cleven $^{1 *}$, Janina Krell-Roesch ${ }^{1,2}$, Claudio R. Nigg ${ }^{1,3}$ and Alexander Woll
}

\begin{abstract}
Background: A growing body of studies that investigated the longitudinal association between physical activity (PA) and the outcome of incident obesity, coronary heart disease (CHD), diabetes and hypertension has become available in recent years. Thus, the purpose of this systematic review was to provide an update on the association between PA and onset of obesity, CHD, diabetes and hypertension in individuals aged $\geq 18$ years who were free of the respective conditions at baseline.

Methods: We systematically searched OVID, Pubmed, and Web of Science databases for pertinent literature published between January of 2012 and February of 2019. To ensure that conclusions are based on high quality evidence, we only included longitudinal studies conducted in samples of $\geq 500$ participants and with $\geq 5$ years of follow-up.

Result: The search yielded 8929 records of which 26 were included in this review. Three studies were conducted on the outcome of incident obesity, eight on incident CHD, nine on incident diabetes, four on incident hypertension, one on the outcome of both diabetes and hypertension, and one on the outcome of CHD, diabetes and hypertension. Overall, there was an association between PA and lower risk of incident obesity, CHD and diabetes, but not hypertension. Higher levels or amount of PA were associated with a reduced risk of new onset of the respective diseases in 20 studies (77\%). Whereas four studies reported an elevated risk of incidence of diseases with lower PA levels (15\%). PA was not associated with incidence of diseases in two studies (8\%).

* Correspondence: laura.cleven@kit.edu

${ }^{1}$ Institute of Sports and Sports Science, Karlsruhe Institute of Technology, Karlsruhe, Germany

Full list of author information is available at the end of the article

(c) The Author(s). 2020 Open Access This article is licensed under a Creative Commons Attribution 4.0 International License, which permits use, sharing, adaptation, distribution and reproduction in any medium or format, as long as you give appropriate credit to the original author(s) and the source, provide a link to the Creative Commons licence, and indicate if changes were made. The images or other third party material in this article are included in the article's Creative Commons licence, unless indicated otherwise in a credit line to the material. If material is not included in the article's Creative Commons licence and your intended use is not permitted by statutory regulation or exceeds the permitted use, you will need to obtain permission directly from the copyright holder. To view a copy of this licence, visit http://creativecommons.org/licenses/by/4.0/. The Creative Commons Public Domain Dedication waiver (http://creativecommons.org/publicdomain/zero/1.0/) applies to the data made available in this article, unless otherwise stated in a credit line to the data. 


\begin{abstract}
(Continued from previous page)
Conclusion: Higher levels of PA are likely associated with a lower risk of becoming obese, develop CHD or diabetes. These findings replicate and strengthen conclusions from earlier reviews underlining the importance of promoting PA in adults. The associations between PA and incident hypertension were less consistent. More research, particularly using prospective cohort designs in large population-based samples, is needed to further untangle the association between PA and incident hypertension.
\end{abstract}

Trail registration: CRD42019124474 (PROSPERO Protocol registration). Date of registration in PROSPERO 27 February 2019.

Keywords: Physical activity, Obesity, Coronary heart disease, Diabetes, Hypertension, Adults, Longitudinal study, Cohort study

\section{Background}

The World Health Organization identified noncommunicable diseases (NCDs), such as diabetes mellitus or cardiovascular diseases to be a major threat to economies and societies [1]. NCDs are implicated in $73 \%$ of all global deaths in 2017 , with 28.8 million deaths attributed to risk factors like high blood pressure, high blood glucose, or high body mass index (BMI) [2]. Furthermore, NCDs are forecasted to account for $81 \%$ of all global deaths in 2040 [3].

NCDs usually develop over a long time period and may be impacted by an individual's health behaviors [4]. As such, many NCDs may be preventable by decreasing metabolic risk factors such as hypertension, overweight and obesity, or hyperglycemia, as well as by decreasing behavioral risk factors like tobacco or alcohol use, an unhealthy diet, and physical inactivity $[1,5]$.

A growing body of research suggests that high levels of physical activity (PA) may have a protective effect on various health conditions including but not limited to overweight and obesity [6], coronary heart disease (CHD) [7, 8], type 2 diabetes mellitus [9, 10], hypertension [11, 12], and hyperglycemia [13, 14]. In addition, several longitudinal studies have become available that examine the association between PA and new onset of NCDs [15-20].

The current systematic review presents an update of a previously published review by our group [20], that examined the long-term effects of PA on type 2 diabetes mellitus, CHD, overweight/obesity and dementia by including studies published before 2012. Given the high significance of this topic and since we expected a substantial amount of relevant studies published after 2012, we provide an updated review of longitudinal studies on the association between PA and incident obesity, CHD, diabetes and hypertension over the past 7 years. Hypertension has been added to the current review as it is widely regarded as a major risk factor for several NCDs [21].

\section{Methods}

This review was conducted in accordance with the Preferred Reporting Items for Systematic Reviews and
Meta-Analysis (PRISMA) guideline [22]. The protocol was registered in the PROSPERO register of systematic reviews (CRD42019124474).

\section{Search strategy}

Pertinent articles published between January of 2012 and February of 2019 were searched in electronic databases (PubMed, Web of Science and EMBASE by OVID) by applying a combination of one or more of the following search terms: "longitudinal and/or long-term"; "physical activity/exercise"; "adult"; "overweight and/or obesity", "coronary heart disease and/or coronary artery disease and/or ischemic disease", "diabetes mellitus and/or diabetes type 2", "hypertension and/or blood pressure". Both titles and abstracts were searched. After identification of studies, their bibliographies were searched manually to identify additional relevant studies.

\section{Study inclusion \& exclusion criteria}

We defined the following inclusion criteria for this systematic review: (1) Longitudinal, i.e. prospective cohort study design; (2) Studies reporting the association between PA and new onset of obesity, CHD, type 2 diabetes mellitus and/or hypertension; (3) Studies providing information on the assessment of PA (predictor variable). PA could be leisure-time/habitual PA, workrelated $\mathrm{PA}$, transportation related $\mathrm{PA}$, organized and unorganized PA, etc.; (4) Only studies with $\geq 5$ years of follow-up were included to allow for a meaningful conclusion on the longitudinal association between PA and selected outcomes of interest; (5) Studies with males and females aged $\geq 18$ years, that were free of the diseases of interest at baseline; (6) Studies with more than 500 participants were included, to improve the probability to capture a substantial amount of incidence cases; and (7) articles written in English.

Excluded from this review were (1) studies investigating the effect of a specific PA intervention, as well as (2) clinical trials, cross-sectional studies, systematic reviews and meta-analyses. 


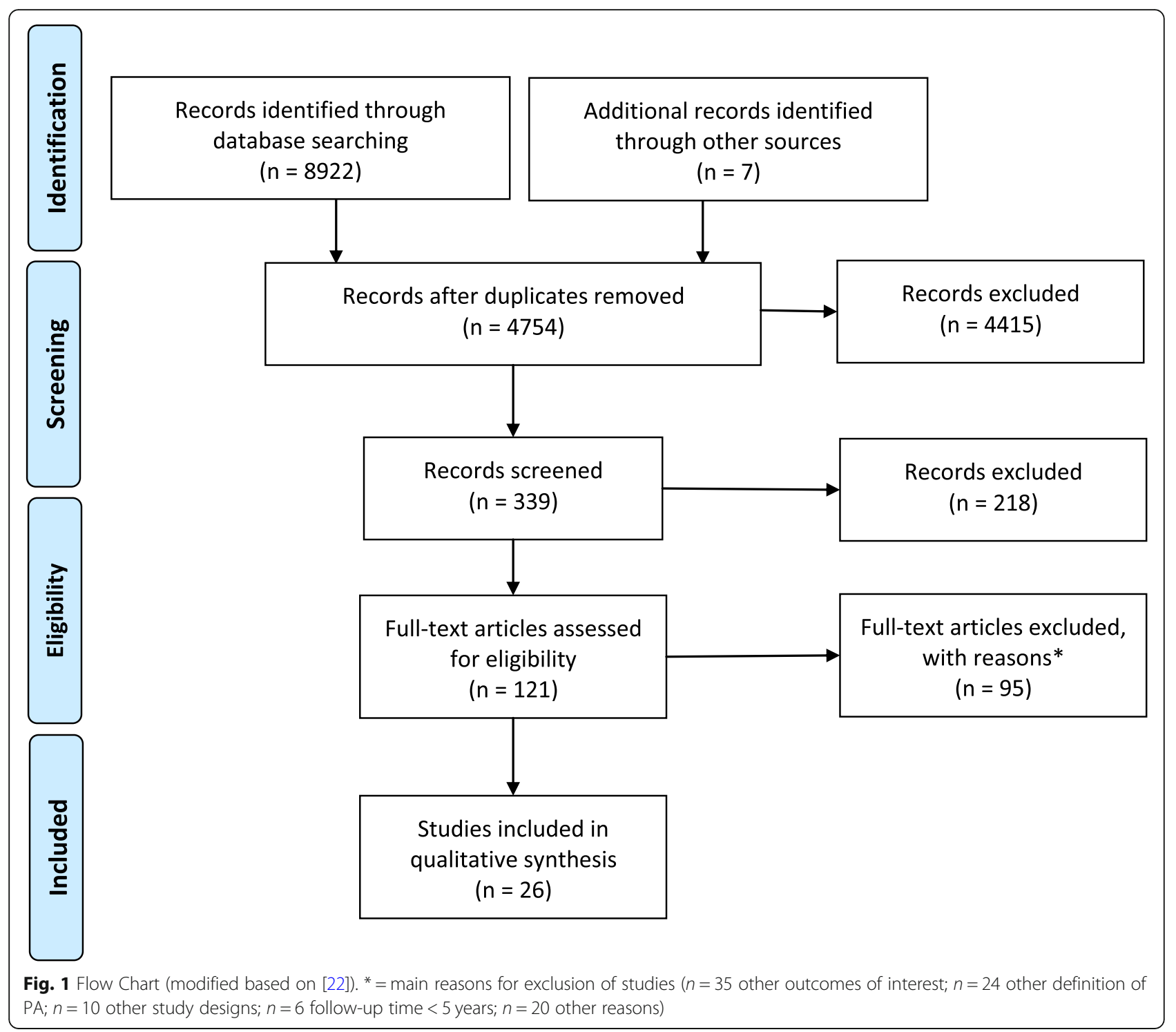

\section{Screening \& data extraction}

All pertinent studies detected after searching the electronic databases were imported to a reference manager software (Citavi 6) and duplicates were removed. The study selection process was divided into three phases. Two independent reviewers (LC \& JKR) screened the titles of the articles, followed by the abstracts, and finally the full-texts based on the inclusion criteria. All studies meeting the eligibility criteria were included in this review. Disagreement was resolved by consensus or by consulting a third author $(\mathrm{CN})$. The following information was extracted by one reviewer (LC): first author's name, publication year, study design, study setting, sample size, follow-up time, participant characteristics (e.g. age, sex, BMI) at baseline, assessment and type of PA (e.g. type, duration, intensity), assessment and type of outcomes of interest (i.e. obesity, CHD, diabetes and hypertension), and main results/ findings of the study (e.g. hazard ratios, relative risk). Extracted data were verified by another author (JKR).

\section{Quality assessment and risk of bias}

The quality of included studies was evaluated independently by two authors (LC \& JKR) using the 22-item Strengthening the Reporting of Observational Studies in Epidemiology (STROBE) statement version 4 [23]. Similarly, potential risk of bias of each study included in this systematic review was assessed through the Tool to Assess Risk of Bias in Cohort Studies [24] by the same authors (LC \& JKR). Any discrepancies between the two reviewers were resolved by discussion or by consulting a third reviewer $(\mathrm{CN})$. 


\section{Results}

Overall, we identified 8929 articles, of which 8903 articles were excluded as they did not meet the inclusion criteria as described above. The reader is referred to Fig. 1 for a flow chart summarizing the search process and number of studies at each step. Twenty-six studies were included in this review with a combined $\mathrm{N}$ of 1,145,298 participants, and follow-up times ranging between 5 and 34 years. Three articles examined the association between PA and incident obesity [25-27], eight studies examined the association between PA and incident CHD [28-35], nine studies examined the association between PA and incident diabetes [36-44], and four studies examined the association between PA and incident hypertension [45-48]. In addition, one study reported the association between both PA and diabetes as well as PA and hypertension [49], and another study reported the association between PA and CHD, diabetes, and hypertension [50].

\section{Association between PA and obesity}

The studies included in this review showed an overall association between higher PA and lower risk of incident obesity. Two out of three studies reported a reduced risk of becoming obese for individuals with high PA levels as compared to low PA $[25,27]$. One study showed an elevated risk (142\%) of becoming obese for persons who were physically inactive [26]. The characteristics of the included studies are summarized in Table 1.

\section{Association between PA and CHD}

Overall, there was an association between higher levels or amount of PA and a decreased risk of incident CHD. Seven out of nine studies reported a reduced risk of new onset of CHD with increasing PA levels as compared to low or no PA [28, 32-35], whereas one study revealed an association between PA and decreased CHD risk only for vigorous intensity PA [29]. One study did not find a significant association for occupational PA and CHD risk [30]. Two out of nine studies examined the impact of change in PA levels over time as predictor variable and failed to detect a significant association with incident CHD [31, 33]. Please refer to Table 2 for a summary of studies on PA and incident CHD.

\section{Association between PA and diabetes}

The studies included in this review provide evidence of an association between increasing PA levels and a decreased risk of incident diabetes. Nine out of 11 studies reported a gradual inverse association between increasing PA levels with up to high/vigorous-intensity and a decreased risk of incident diabetes [36-39,

Table 1 Overview of longitudinal studies on the association between PA and the outcome of obesity $\left(\mathrm{BMl} \geq 30 \mathrm{~kg} / \mathrm{m}^{2}\right)$

\begin{tabular}{|c|c|c|c|c|c|c|}
\hline Author & Country & Characteristics & Follow-up time & $\begin{array}{l}\text { Predictor variable: Physical } \\
\text { activity }\end{array}$ & $\begin{array}{l}\text { Outcome } \\
\text { of interest }\end{array}$ & Main results \\
\hline \multirow[t]{4}{*}{$\begin{array}{l}\text { Bell et al. } \\
(2014) \text { [25] }\end{array}$} & \multirow{4}{*}{$\begin{array}{l}\text { UK, } \\
\text { Whitehall } \\
\text { II study }\end{array}$} & \multirow[t]{4}{*}{$\begin{array}{l}N=3670,73 \% \text { male, } \\
55.5 \pm 6.0 \text { years }\end{array}$} & \multirow{4}{*}{$\begin{array}{l}10 \text { years; Baseline } \\
\text { 1997-1999 } \\
\text { Follow-up: 2002-- } \\
\text { 2004, 2007-2009 }\end{array}$} & $\begin{array}{l}\text { Self-reported, duration of } \\
\text { MVPA (h/wk) }\end{array}$ & \multirow[t]{4}{*}{$\begin{array}{l}\text { Incident } \\
\text { obesity }\end{array}$} & $\begin{array}{l}\text { OR }[95 \% \mathrm{Cl}] \text { : low level PA as } \\
\text { reference }{ }^{\text {a: }}\end{array}$ \\
\hline & & & & -Low: 0-1.5 & & -High level PA $0.64[0.44,0.93]$ \\
\hline & & & & -Intermediate: 1.56-4.25 & & after 5 years \\
\hline & & & & -High: 4.27-20.56 & & $\begin{array}{l}\text {-High level PA } 0.63[0.45,0.88] \\
\text { after } 10 \text { years }\end{array}$ \\
\hline \multirow[t]{3}{*}{$\begin{array}{l}\text { Montgomerie } \\
\text { et al. (2014) } \\
{[26]}\end{array}$} & \multirow[t]{3}{*}{ Australia } & \multirow[t]{3}{*}{$\begin{array}{l}N=1521,50.6 \% \\
\text { male, age } 44.6 \pm \\
16.22 \text { years }\end{array}$} & \multirow{3}{*}{$\begin{array}{l}2898.9 \pm 402.29 \text { days } \\
\text { Baseline: 1999-2003, } \\
\text { follow-up: } 2004- \\
\text { 2006, 2008-2010 }\end{array}$} & $\begin{array}{l}\text { Self-reported, score: } \\
\text { frequency } x \text { time per } \\
\text { session } x \text { intensity }\end{array}$ & \multirow[t]{3}{*}{$\begin{array}{l}\text { Incident } \\
\text { obesity }\end{array}$} & $\begin{array}{l}\text { RR }[95 \% \text { CI]: Association between } \\
\text { physical inactivity \& incident } \\
\text { obesity }^{\text {b. }}\end{array}$ \\
\hline & & & & $\begin{array}{l}\text {-Inactive: }<100 \text { sedentary, } \\
\text { 100-1600 low }\end{array}$ & & $-1.42[1.03,1.95] p=0.030$ \\
\hline & & & & $\begin{array}{l}\text {-Active: 1600-3200 } \\
\text { moderate, > } 3200 \text { high }\end{array}$ & & \\
\hline \multirow[t]{6}{*}{$\begin{array}{l}\text { Pavey et al. } \\
\text { (2016) [27] }\end{array}$} & \multirow[t]{6}{*}{ Australia } & \multirow{6}{*}{$\begin{array}{l}N=2735 \text { women, } \\
24.6(20.6-28.5) \\
\text { years }\end{array}$} & \multirow{6}{*}{$\begin{array}{l}12 \text { years } \\
\text { Baseline: } \\
2000 \\
\text { Follow-up: } 2012\end{array}$} & $\begin{array}{l}\text { Self-reported, } \\
\text { score (MET-min/wk) }\end{array}$ & \multirow{6}{*}{$\begin{array}{l}\text { Change } \\
\text { in BMI } \\
\text { category }\end{array}$} & $\begin{array}{l}\text { OR }[95 \% \mathrm{Cl}] \text { : increasing cumulative } \\
\text { PA with very low activity as }\end{array}$ \\
\hline & & & & -Very low $(<250)$ & & rete \\
\hline & & & & -Low $(250$ to $<500)$ & & $\begin{array}{l}\text {-Transition to obesity: } 0.73[0.59 \\
0.90], p<.05\end{array}$ \\
\hline & & & & -Active $(500$ to $<1000)$ & & OR [95\% Cl], very high cumulative \\
\hline & & & & -Very active (> 1000) & & $\begin{array}{l}\text { PA with very low activity as } \\
\text { referencec: }\end{array}$ \\
\hline & & & & & & $\begin{array}{l}\text {-Transition to obesity } 0.52[0.30 \\
0.92], p<.05\end{array}$ \\
\hline
\end{tabular}

Abbreviation: BMI Body Mass Index, $C l$ confidence interval, $h$ hour, MET metabolic equivalent, min minutes, MVPA moderate-to-vigorous physical activity, $N$ number of participants, $O R$ odds ratio, $p p$-value, $P A$ physical activity, $R R$ relative risk, $S D$ Standard deviation, wk week

${ }^{a}$ Model adjusted for age, sex, ethnicity; ${ }^{\text {b }}$ Model adjusted for age, sex, chronic conditions (diabetes, asthma, chronic obstructive pulmonary disease, cardiovascular disease and mental health); ${ }^{c}$ Model adjusted for educational level, area of residence, number of children, occupation, work time walking, work time in heavy labor, smoking status, alcohol consumption, energy intake, dieting, oral contraceptive pill use, number of chronic conditions 
Table 2 Overview of longitudinal studies on the association between PA and the outcome of CHD

\begin{tabular}{|c|c|c|c|c|c|c|}
\hline Author & Country & Characteristics & $\begin{array}{l}\text { Follow- } \\
\text { up time }\end{array}$ & $\begin{array}{l}\text { Predictor variable: Physical } \\
\text { activity }\end{array}$ & $\begin{array}{l}\text { Outcome of } \\
\text { interest }\end{array}$ & Main results \\
\hline \multirow{8}{*}{$\begin{array}{l}\text { Chomistek } \\
\text { et al. } \\
\text { (2016) [28] }\end{array}$} & \multirow{8}{*}{$\begin{array}{l}\text { USA, Nurses' } \\
\text { Health Study II } \\
\text { (NHSII) }\end{array}$} & \multirow[t]{8}{*}{$\begin{array}{l}N=97,230 \text { women, } 36.6 \pm \\
4.6 \text { years }\end{array}$} & \multirow{3}{*}{$\begin{array}{l}20 \text { years } \\
\text { Baseline: } \\
1991\end{array}$} & \multirow{3}{*}{$\begin{array}{l}\text { Self-reported LTPA } \\
\text { (MET-h/wk, in quintiles) } \\
-<1\end{array}$} & \multirow{8}{*}{$\begin{array}{l}\text { Incident CHD } \\
\text { (nonfatal Ml, fatal } \\
\text { CHD) }\end{array}$} & $\begin{array}{l}\text { HR [95\% Cl] of CHD event } \\
\text { for total LTPA: }\end{array}$ \\
\hline & & & & & & $-<1: 1.0$ (reference) \\
\hline & & & & & & - 1-5.9: $0.86[0.68,1.08]$ \\
\hline & & & \multirow{5}{*}{$\begin{array}{l}\text { Follow- } \\
\text { up: } 2011\end{array}$} & $-1-5.9$ & & \multirow[t]{2}{*}{ - 6-14.9: $0.66[0.52,0.84]$} \\
\hline & & & & $-6-14.9$ & & \\
\hline & & & & $-15-29.9$ & & - 15-29.9: $0.48[0.36,0.63]$ \\
\hline & & & & $-\geq 30$ & & $-\geq 30: 0.53[0.41,0.70]$ \\
\hline & & & & & & $\begin{array}{l}\text { Similarly, increasing MET-h/ } \\
\text { wk were associated with a } \\
\text { decreased risk of incident } \\
\text { CHD when looking at } \\
\text { moderate-intensity PA only, } \\
\text { as well as looking at } \\
\text { vigorous-intensity PA only. }\end{array}$ \\
\hline \multirow{3}{*}{$\begin{array}{l}\text { Delaney } \\
\text { et al. } \\
\text { (2013) [29] }\end{array}$} & \multirow{3}{*}{$\begin{array}{l}\text { USA, Multi- } \\
\text { Ethnic Study of } \\
\text { Atherosclerosis } \\
\text { (MESA) }\end{array}$} & \multirow{3}{*}{$\begin{array}{l}N=5656,47.4 \% \text { male, } 61.3 \pm \\
9.9 \text { years, BMl } 28.3 \pm 5.4 \\
\mathrm{~kg} / \mathrm{m}^{2}\end{array}$} & \multirow{2}{*}{$\begin{array}{l}5 \text { years } \\
\text { Baseline } \\
2000- \\
2002\end{array}$} & \multirow[t]{3}{*}{$\begin{array}{l}\text { Self-reported PA (total } \\
\mathrm{min} / \mathrm{d} \text {, total MET-min/d) }\end{array}$} & \multirow[t]{3}{*}{ Incident CAC } & $\begin{array}{l}\text { RR }[95 \% \mathrm{Cl}] \text { of } \mathrm{PA} \text { and } \\
\text { incident } \mathrm{CAC} \text { : }\end{array}$ \\
\hline & & & & & & $\begin{array}{c}\text { - Vigorous activity: } 0.97 \\
{[0.94,1.00], p=0.048}\end{array}$ \\
\hline & & & $\begin{array}{l}\text { Follow- } \\
\text { up: } \\
2005- \\
2007\end{array}$ & & & $\begin{array}{l}\text { No association between } \\
\text { intentional, sedentary, } \\
\text { MVPA and conditioning PA } \\
\text { and incident CAC. }\end{array}$ \\
\hline \multirow[t]{9}{*}{$\begin{array}{l}\text { Ferrario } \\
\text { et al. } \\
(2018)[30]\end{array}$} & \multirow[t]{9}{*}{$\begin{array}{l}\text { Italy, MONICA, } \\
\text { PAMELA, SEMM }\end{array}$} & \multirow[t]{9}{*}{$N=3574$ men, $25-64$ years } & $\begin{array}{l}\text { Median } \\
14 \text { years } \\
\text { (IQR }\end{array}$ & \multirow{4}{*}{$\begin{array}{l}\text { Self-reported, OPA score } \\
1-5 \text { (tertiles, cut-offs at } 2.5, \\
3.125 \text { ), SpPA index (min/wk } \\
\text { of MPA or VPA based on } \\
\text { METs of task) }\end{array}$} & \multirow{9}{*}{$\begin{array}{l}\text { Incident CHD (first } \\
\text { acute coronary } \\
\text { event as MI, acute } \\
\text { coronary } \\
\text { syndrome or } \\
\text { coronary } \\
\text { revascularization) }\end{array}$} & $\begin{array}{l}\text { HR }[95 \% \mathrm{Cl} \text { ] of first CHD } \\
\text { event (fatal or non-fatal) by } \\
\text { OPA group }{ }^{\text {: }}\end{array}$ \\
\hline & & & $\begin{array}{l}12.9- \\
15.9)\end{array}$ & & & - Low: $1.66[1.06,2.59]$ \\
\hline & & & \multirow{2}{*}{$\begin{array}{l}\text { Baseline: } \\
1989- \\
1996\end{array}$} & & & $\begin{array}{l}\text { - Intermediate: } 1.0 \\
\text { (reference) }\end{array}$ \\
\hline & & & & & & - High: 1.18 [0.72, 1.94] \\
\hline & & & \multirow{5}{*}{$\begin{array}{l}\text { Follow- } \\
\text { up: } 2008\end{array}$} & - Poor: 0 & & \multirow{2}{*}{$\begin{array}{l}\text { HR [95\% CI] of first CHD } \\
\text { event (fatal or non-fatal) by } \\
\text { SpPA groupc: }\end{array}$} \\
\hline & & & & \multirow{3}{*}{$\begin{array}{l}\text { - Intermediate: 1-149 } \\
\text { MPA or 1-74 VPA or } \\
\text { 1-149 MPA plus VPA }\end{array}$} & & \\
\hline & & & & & & - Poor: 1.0 (reference) \\
\hline & & & & & & $\begin{array}{l}\text { - Intermediate: } 0.81 \text { [0.50, } \\
1.32]\end{array}$ \\
\hline & & & & $\begin{array}{l}\text { - Recommended: } \geq 150 \\
\text { MPA or } \geq 75 \text { VPA or } \\
\geq 150 \text { MPA plus VPA }\end{array}$ & & $\begin{array}{l}\text { - Recommended: } 0.58 \text { [0.30, } \\
1.12]\end{array}$ \\
\hline \multirow{9}{*}{$\begin{array}{l}\text { Jefferis } \\
\text { et al. } \\
\text { (2014) [31] }\end{array}$} & \multirow{9}{*}{$\begin{array}{l}\text { UK, British } \\
\text { Regional Heart } \\
\text { Study }\end{array}$} & \multirow[t]{9}{*}{$\begin{array}{l}N=3320 \text { men, } 68.3 \pm 5.4 \\
\text { years }\end{array}$} & \multirow{9}{*}{$\begin{array}{l}\text { Median } \\
11 \text { years } \\
\text { Baseline } \\
1998- \\
2000 \\
\text { Follow- } \\
\text { up: } 2010\end{array}$} & $\begin{array}{l}\text { Self-reported usual PA } \\
\text { (score) }\end{array}$ & \multirow{9}{*}{$\begin{array}{l}\text { Incident CHD (First } \\
\text { fatal or nonfatal MI } \\
\text { events, ICD-9 Code } \\
410-414, \text { ICD - } 10 \\
\text { Code I21-I23, I252) }\end{array}$} & \multirow{2}{*}{$\begin{array}{l}\text { HR }[95 \% \mathrm{Cl} \text { ) of first CHD } \\
\text { event (fatal or non-fatal) by } \\
\text { PA group }{ }^{d} \text { : }\end{array}$} \\
\hline & & & & - Inactive (0-2) & & \\
\hline & & & & - Occasional (3-5) & & - None: 1.0 (reference) \\
\hline & & & & - Light (6-8) & & $\begin{array}{l}\text { - Occasional: } 0.52 \text { [0.34, } \\
0.79]\end{array}$ \\
\hline & & & & - Moderate (9-12) & & - Light: 0.47 [0.30, 0.74] \\
\hline & & & & $\begin{array}{l}\text { - Moderately vigorous (13- } \\
\text { 20) }\end{array}$ & & - Moderate: $0.51[0.32,0.82]$ \\
\hline & & & & $\begin{array}{l}\text { 20) } \\
\text { - Vigorous (> 21) }\end{array}$ & & $\begin{array}{l}\text { - Moderately vigorous and } \\
\text { vigorous: } 0.44[0.29,0.65]\end{array}$ \\
\hline & & & & $\begin{array}{l}\text { Change in PA } \\
(1996-2000)\end{array}$ & & $p=0.004$ \\
\hline & & & & - Always inactive & & HR [95\% Cl] of first CHD \\
\hline
\end{tabular}


Table 2 Overview of longitudinal studies on the association between PA and the outcome of CHD (Continued)

\begin{tabular}{|c|c|c|c|c|c|c|}
\hline Author & Country & Characteristics & $\begin{array}{l}\text { Follow- } \\
\text { up time }\end{array}$ & $\begin{array}{l}\text { Predictor variable: Physical } \\
\text { activity }\end{array}$ & $\begin{array}{l}\text { Outcome of } \\
\text { interest }\end{array}$ & Main results \\
\hline & & & & - Became inactive & & $\begin{array}{l}\text { event (fatal or non-fatal) by } \\
\text { change in PA group }{ }^{d} \text { : }\end{array}$ \\
\hline & & & & - Became active & & $\begin{array}{l}\text { - Always inactive: } 1.0 \\
\text { (reference) }\end{array}$ \\
\hline & & & & \multirow[t]{3}{*}{ - Always active } & & $\begin{array}{l}\text { - Became inactive: } 0.87 \\
{[0.53,1.45]}\end{array}$ \\
\hline & & & & & & $\begin{array}{l}\text { - Became active: } 0.86 \text { [0.55, } \\
1.35]\end{array}$ \\
\hline & & & & & & $\begin{array}{l}\text { - Always active: } 0.73[0.53, \\
1.02]\end{array}$ \\
\hline \multirow[t]{6}{*}{$\begin{array}{l}\text { Koolhaas } \\
\text { et al. } \\
(2016) \text { [32] }\end{array}$} & \multirow[t]{6}{*}{$\begin{array}{l}\text { Netherlands, } \\
\text { The Rotterdam } \\
\text { Study }\end{array}$} & \multirow[t]{6}{*}{$\begin{array}{l}N=5901, \text { median age } 67 \\
\text { years }\end{array}$} & \multirow{6}{*}{$\begin{array}{l}15 \text { years } \\
\text { Baseline } \\
1997- \\
2001 \\
\text { Follow- } \\
\text { up: } 2012\end{array}$} & \multirow[t]{2}{*}{$\begin{array}{l}\text { Self-reported PA, tertiles } \\
\text { (median (range) } \\
\text { MET-h/wk in total PA) }\end{array}$} & \multirow{6}{*}{$\begin{array}{l}\text { Incident CHD (fatal } \\
\text { or nonfatal MI, } \\
\text { surgical/ } \\
\text { percutaneous } \\
\text { coronary } \\
\text { revascularization } \\
\text { procedure) }\end{array}$} & $\begin{array}{l}\text { Total PA and risk of } \\
\text { incident CHD event } \mathrm{e}^{\mathrm{H}} \text { HR } \\
{[95 \% \mathrm{CI}]}\end{array}$ \\
\hline & & & & & & - Tertile 1: 1.0 (reference) \\
\hline & & & & \multirow{2}{*}{$\begin{array}{l}\text { - Tertiles } 1: 42.0(\leq 61.4) \hat{=} \\
\quad 1.5 \mathrm{~h} / \mathrm{d} \text { at } 4 \mathrm{METs}\end{array}$} & & - Tertile 2: $0.76[0.63,0.92]$ \\
\hline & & & & & & - Tertile 3:0.69 [0.57, 0.84] \\
\hline & & & & $\begin{array}{l}\text { - Tertiles 2: } 77.5 \text { (61.5-96.9) } \\
\quad \text { = } 2.8 \mathrm{~h} / \mathrm{d} \text { at } 4 \text { METs }\end{array}$ & & \multirow{2}{*}{$\begin{array}{l}\text { Per } 10 \text { MET-h/wk: } 0.96 \\
{[0.94,0.98] . \text { p overall }=<} \\
0.001 .\end{array}$} \\
\hline & & & & $\begin{array}{l}\text { - Tertiles 3: } 126.7(\geq 97.0) \\
\quad \quad 1.5 \mathrm{~h} / \mathrm{d} \text { at } 4 \mathrm{METs}\end{array}$ & & \\
\hline \multirow{17}{*}{$\begin{array}{l}\text { Petersen } \\
\text { et al. } \\
\text { (2012) [33] }\end{array}$} & \multirow{17}{*}{$\begin{array}{l}\text { Denmark, } \\
\text { Copenhagen } \\
\text { City Heart } \\
\text { Study }\end{array}$} & \multirow{17}{*}{$\begin{array}{l}N=10,443,57 \% \text { female, } \\
\text { median age } 58 \text { years }\end{array}$} & \multirow{2}{*}{$\begin{array}{l}\text { Baseline } \\
\text { 1976-78 }\end{array}$} & Self-reported LTPA & \multirow{17}{*}{$\begin{array}{l}\text { Incident CHD fatal } \\
\text { and non-fatal cases } \\
\text { (MI: ICD-8 Code } \\
410 \text {, ICD-10 Code } \\
\text { I21-22; IHD: ICD-8 } \\
\text { Code 410-414, } \\
\text { ICD-10 Code I20- } \\
\text { 25) }\end{array}$} & $\mathrm{HR}[95 \% \mathrm{Cl}]$ of IHD by PA \\
\hline & & & & - Sedentary & & $\begin{array}{l}\text { Change: } \\
\text { Women | Men }\end{array}$ \\
\hline & & & \multirow{15}{*}{$\begin{array}{l}\text { Follow- } \\
\text { up: } \\
1981- \\
1983 \\
2008\end{array}$} & - Light & & $--2: 1.60[1.02,2.32] \mid 1.33$ \\
\hline & & & & -Moderate & & {$[0.97,1.83]$} \\
\hline & & & & - Vigorous & & $--1: 1.28[1.10,1.49] \mid 1.12$ \\
\hline & & & & Change in PA categories & & {$[0.96,1.31]$} \\
\hline & & & & $(1976 / 78-1981 / 83)$ & & - 0: 1.0 (reference) \\
\hline & & & & - $-2 /-3$ categories & & - 1: $0.97[0.85,1.12] \mid 1.09$ \\
\hline & & & & - -1 category & & \\
\hline & & & & - 0 (stable) & & - 2: $1.01[0.75,1.38] \mid 1.16$ \\
\hline & & & & -+1 category & & \\
\hline & & & & $-+2 /+3$ categories & & $\begin{array}{l}\mathrm{HR}[95 \% \mathrm{Cl}] \text { of } \mathrm{Ml} \text { by PA } \\
\text { change } \mathrm{f}^{\mathrm{f}} \\
\text { Women | Men }\end{array}$ \\
\hline & & & & & & $\begin{array}{l}--2: 1.56[0.89,2.75] \mid 1.74 \\
{[1.17,2.60]}\end{array}$ \\
\hline & & & & & & $\begin{array}{l}--1: 1.30[1.03,1.65] \mid 1.13 \\
{[0.91,1.39]}\end{array}$ \\
\hline & & & & & & - 0: 1 (reference) \\
\hline & & & & & & $\begin{array}{l}-1: 0.98[0.79,1.22] \mid 1.14 \\
{[0.95,1.36]}\end{array}$ \\
\hline & & & & & & $\begin{array}{l}-2: 1.08[0.67,1.75] \mid 1.30 \\
{[0.92,1.84]}\end{array}$ \\
\hline $\begin{array}{l}\text { Soares- } \\
\text { Miranda } \\
\text { et al. }\end{array}$ & USA & $\begin{array}{l}N=4207,39 \% \text { males, } 72.5 \pm \\
5.5 \text { years }\end{array}$ & $\begin{array}{l}10 \text { years } \\
\text { Baseline: } \\
1989\end{array}$ & $\begin{array}{l}\text { Self-reported LTPA } \\
\text { (kcal/wk), exercise } \\
\text { intensity }\end{array}$ & $\begin{array}{l}\text { Incident CHD (fatal } \\
\text { \& nonfatal MI \& } \\
\text { CHD death) }\end{array}$ & $\begin{array}{l}\text { HR }[95 \% \mathrm{Cl}] \text { for exercise } \\
\text { intensity }{ }^{9} \text {, none as } \\
\text { reference: }\end{array}$ \\
\hline (2016) [34] & & & $\begin{array}{l}\text { Follow- } \\
\text { up: } 1999\end{array}$ & - None & & $\begin{array}{l}\text { - Low: } 0.56[0.43,0.72], p< \\
0.001\end{array}$ \\
\hline & & & & - Low & & $\begin{array}{l}\text { - Moderate: } 0.53[0.41,0.69] \text {, } \\
\quad p<0.001\end{array}$ \\
\hline
\end{tabular}


Table 2 Overview of longitudinal studies on the association between PA and the outcome of CHD (Continued)

\begin{tabular}{|c|c|c|c|c|c|c|}
\hline Author & Country & Characteristics & $\begin{array}{l}\text { Follow- } \\
\text { up time }\end{array}$ & $\begin{array}{l}\text { Predictor variable: Physical } \\
\text { activity }\end{array}$ & $\begin{array}{l}\text { Outcome of } \\
\text { interest }\end{array}$ & Main results \\
\hline & & & & - Moderate & & - High: 0.47 [0.32, 0.69], \\
\hline & & & & - High & & \\
\hline \multirow[t]{2}{*}{$\begin{array}{l}\text { Tikkanen } \\
\text { et al. } \\
\text { (2018) [35] }\end{array}$} & \multirow[t]{2}{*}{ UK } & \multirow[t]{2}{*}{$\begin{array}{l}N=502,635,54 \% \text { female, } \\
56.5 \pm 8.1 \text { years }\end{array}$} & $\begin{array}{l}\text { Median } \\
6.1 \text { years } \\
\text { Baseline: } \\
2006-10\end{array}$ & Self-reported (MET-h/wk) & \multirow[t]{2}{*}{$\begin{array}{l}\text { Incident CHD (ICD- } \\
9 \text { Code 410-411, } \\
\text { ICD-10 Code I20.0, } \\
\text { I21, I22) }\end{array}$} & \multirow[t]{2}{*}{$\begin{array}{l}\text { HR }[95 \% \mathrm{Cl} \text { ] association } \\
\text { CHD and PA level }\left.\right|^{h}: \\
-0.95[0.93,0.97], p<0.001\end{array}$} \\
\hline & & & $\begin{array}{l}\text { Follow- } \\
\text { up: } \\
2015- \\
2016\end{array}$ & & & \\
\hline \multirow[t]{6}{*}{$\begin{array}{l}\text { Williams \& } \\
\text { Thompson } \\
\text { (2012) [50] }\end{array}$} & \multirow[t]{6}{*}{ USA } & \multirow[t]{6}{*}{$N=47,921$} & $\begin{array}{l}\text { Median } \\
6.2 \text { years }\end{array}$ & $\begin{array}{l}\text { Self-reported PA MET-h/d } \\
\text { - Light }\end{array}$ & \multirow{6}{*}{$\begin{array}{l}\text { Incident CHD (MI, } \\
\text { CABG, } \\
\text { percutaneous } \\
\text { coronary } \\
\text { intervention, and } \\
\text { angina pectoris) }\end{array}$} & $\begin{array}{l}\text { Greater MET-h/d is associ- } \\
\text { ated with lower risk of inci- } \\
\text { dent CHD: HR }[95 \% \mathrm{Cl}]^{\mathrm{i}}\end{array}$ \\
\hline & & & \multirow{2}{*}{$\begin{array}{l}\text { Baseline: } \\
\text { 1998- } \\
1999\end{array}$} & - Moderate & & - Running: $0.955[0.91,1.00]$ \\
\hline & & & & - Vigorous & & $\begin{array}{l}\text { - Walking: } 0.907 \text { [0.839, } \\
0.98]\end{array}$ \\
\hline & & & \multirow[t]{3}{*}{$\begin{array}{l}\text { Follow- } \\
\text { up: } 2006\end{array}$} & & & $\begin{array}{l}\text { - Other vigorous: } 0.99 \\
{[0.966,1.02]}\end{array}$ \\
\hline & & & & & & $\begin{array}{l}\text { - Other moderate: } 0.98 \\
{[0.927,1.04]}\end{array}$ \\
\hline & & & & & & $\begin{array}{l}\text { - Other light: } 0.98 \text { [0.807, } \\
\text { 1.197] }\end{array}$ \\
\hline
\end{tabular}

Abbreviation: $B M I$ Body Mass Index, $C A B G$ coronary artery bypass graphs, $C A C$ coronary artery calcification, $C H D$ coronary heart disease, $C I$ confidence interval, $d$ day, $h$ hour, $H D L$ high density lipoprotein, HR hazard ratio, ICD International Classification of Diseases, IHD ischemic heart disease, IQR interquartile range, $k c a l$ kilocalories, LTPA leisure time physical activity, MET metabolic equivalent, MI Myocardial Infarction, min minutes, MPA moderate physical activity, MVPA moderate to vigorous physical intensity, $N$ number of participants, $O P A$ occupational physical activity, $p$ p-value, $P A$ physical activity, $R R$ relative risk, $S p P A$ sport physical activity, VPA vigorous physical activity, wk week

${ }^{\mathrm{a}}$ Model adjusted for age; ${ }^{\mathrm{b}}$ Model adjusted for age, sex, ethnicity, BMI, pack years of smoking, family history of Ml, hypertension, dyslipidemia, diabetes, education, alcohol use, current smoking status, education, income, health insurance status; c: Model adjusted for age, cohort, educational level, OPA and SpPA; $\mathrm{d}_{\text {Model adjusted for age and region; }}{ }^{\mathrm{e}}$ Model adjusted for age and sex; ${ }^{\mathrm{f}}$ Model adjusted for physical activity level in 1976-1978, age, education, smoking habits, alcohol consumption, BMI, diabetes, cholesterol, blood pressure lowering therapy in 1981-1983; ${ }^{9}$ Model adjusted for age, sex, race, education, income, clinical sites, smoking, BMl; ${ }^{h}$ Model adjusted for age, sex, region; ${ }^{i}$ Model adjusted for baseline age (age, age2), sex, race, education, smoking, intakes of red meat, fruit, alcohol

$40-42,44,50]$, whereas one study revealed an association only between moderate intensity PA and reduced diabetes risk [39]. Two out of 11 studies reported an increased risk of incident diabetes (179 and $145 \%$, respectively) for participants engaging in low amount of leisure time PA as compared to the highly active reference group [43, 49]. For a summary of included studies on the association between PA and diabetes please refer to Table 3 .

\section{Association between PA and hypertension}

Overall, there was no consistent association between PA and incident hypertension. Three out of six studies reported a gradual inverse association between PA levels (running and walking, moderate and moderate-vigorous PA) and incident hypertension [46, 48, 50], whereas one study found an association only for a specific age group (51-60 years) [48]. Two out of six studies found no significant association between PA and incident hypertension [45, 47]. One out of six studies reported an increased risk of incident hypertension (137\%) for persons with low leisure time PA as compared to the highly active reference group [49]. Please refer to Table 4 for an overview of included studies.

\section{Discussion}

The purpose of this research was to review studies published after January of 2012 and up to February of 2019 that investigated the long-term association between PA and new onset of obesity, CHD, diabetes and hypertension. Overall, we observed an association between PA and a decreased risk of incident obesity, CHD and diabetes but not hypertension. This is in line with systematic reviews published by our group and others that also found beneficial associations of PA with overweight/obesity, CHD and diabetes [20, 51-53]. Furthermore, it is also consistent with our hypothesis derived from both interventional and observational studies [54-57].

The included studies that examined the association between PA and incident hypertension reported conflicting results. This is partly in accordance with two other meta-analyses [58, 59]. One meta-analysis reported a 
Table 3 Overview of longitudinal studies on the association between PA and the outcome of diabetes

\begin{tabular}{|c|c|c|c|c|c|c|}
\hline Author & Country & Characteristics & $\begin{array}{l}\text { Follow-up } \\
\text { time }\end{array}$ & $\begin{array}{l}\text { Predictor variable: Physical } \\
\text { activity }\end{array}$ & Outcome of interest & Main results \\
\hline \multirow[t]{4}{*}{$\begin{array}{l}\text { Carlsson } \\
\text { et al. } \\
(2013)[36]\end{array}$} & \multirow[t]{4}{*}{$\begin{array}{l}\text { Sweden } \\
\text { (Swedish Twin } \\
\text { Registry) }\end{array}$} & \multirow[t]{4}{*}{$N=23,539$} & \multirow[t]{2}{*}{$\begin{array}{l}\text { Baseline: } \\
\text { 1967-1972 }\end{array}$} & Self-reported LTPA & \multirow[t]{4}{*}{ Incident type 2 diabetes } & $\begin{array}{l}\text { Risk of type } 2 \text { diabetes } \\
\text { decreased with PA: HR } \\
{[95 \% \mathrm{Cl}]}\end{array}$ \\
\hline & & & & - Low & & - Low: 1.0 (reference) \\
\hline & & & \multirow{2}{*}{$\begin{array}{l}\text { Follow-up: } \\
\text { 1998-2002 }\end{array}$} & - Moderate & & - Moderate: 0.77 [0.61, 0.96] \\
\hline & & & & - high & & - High: $0.53[0.37,0.75]$ \\
\hline \multirow{3}{*}{$\begin{array}{l}\text { Elwood } \\
\text { et al. } \\
\text { (2013) [40] }\end{array}$} & \multirow{3}{*}{$\begin{array}{l}\text { UK (Caerphilly } \\
\text { Prospective } \\
\text { Study CaPS) }\end{array}$} & \multirow{3}{*}{$\begin{array}{l}N=2235 \\
\text { men, } 45-59 \\
\text { years }\end{array}$} & 30 years & \multirow[t]{3}{*}{ Self-reported PA } & \multirow[t]{3}{*}{$\begin{array}{l}\text { Incident diabetes (self- } \\
\text { reported) }\end{array}$} & $\begin{array}{l}\text { OR }[95 \% \mathrm{Cl}] \text { for regular activity } \\
\text { and incident diabetes }\end{array}$ \\
\hline & & & $\begin{array}{l}\text { Baseline: } \\
\text { 1979-1983 }\end{array}$ & & & $-0.63[0.46,0.85]$ \\
\hline & & & $\begin{array}{l}\text { Follow-up: } \\
\text { 1984-1988, } \\
\text { 1989-1993, } \\
\text { 1993-1997, } \\
2009\end{array}$ & & & \\
\hline \multirow{10}{*}{$\begin{array}{l}\text { Grøntved } \\
\text { et al. } \\
\text { (2014) [41] }\end{array}$} & \multirow{10}{*}{$\begin{array}{l}\text { USA } \\
\text { (Nurses'Health } \\
\text { Study NHS I } \\
\text { and II) }\end{array}$} & \multirow[t]{10}{*}{$\begin{array}{l}N=99,316 \\
\text { women }\end{array}$} & 8 years & \multirow{5}{*}{$\begin{array}{l}\text { Self-reported PA (time spent } \\
\text { on resistance exercise per } \\
\text { week, lower intensity } \\
\text { muscular conditioning } \\
\text { exercises (yoga, stretching, } \\
\text { toning), aerobic MVPA) }\end{array}$} & \multirow{10}{*}{$\begin{array}{l}\text { Incident diabetes (self- } \\
\text { report confirmed using } \\
\text { standardized criteria; } \\
\text { validated in sub-sample } \\
\text { through medical chart } \\
\text { review) }\end{array}$} & $\begin{array}{l}\text { RR }[95 \% \mathrm{Cl} \text { ] for incident } \\
\text { diabetes for aerobic MVPA }\end{array}$ \\
\hline & & & Baseline & & & - None: 1.0 (reference) \\
\hline & & & $\begin{array}{l}(\mathrm{NHS} \mathrm{I):} \\
2000\end{array}$ & & & - 1-29 min: $0.83[0.74,0.92]$ \\
\hline & & & \multirow{2}{*}{$\begin{array}{l}\text { Baseline } \\
\text { (NHS II): } \\
2001\end{array}$} & & & - 30-59 min: $0.73[0.65,0.82]$ \\
\hline & & & & & & - 60-150 min: $0.66[0.60,0.73]$ \\
\hline & & & \multirow{2}{*}{$\begin{array}{l}\text { Follow-up } \\
\text { (NHS I): } \\
2008\end{array}$} & - None & & $-\geq 150$ min: $0.46[0.41,0.50]$ \\
\hline & & & & $-1-29 \mathrm{~min} / \mathrm{wk}$ & & - Trend: $p<0.001$ \\
\hline & & & \multirow{3}{*}{$\begin{array}{l}\text { Follow-up } \\
\text { (NHS II): } \\
2009\end{array}$} & - 30-59 min/wk & & \multirow{3}{*}{$\begin{array}{l}\text { Engaging in at least } 150 \mathrm{~min} / \mathrm{wk} \\
\text { of aerobic MVPA and at least } \\
60 \mathrm{~min} / \mathrm{wk} \text { of muscle- } \\
\text { strengthening activities was } \\
\text { significantly associated with } \\
\text { lower risk of incident diabetes } \\
\text { compared with being inactive } \\
\text { (pooled RR }=0.33[0.29,0.38] \text { ). }\end{array}$} \\
\hline & & & & $-60-50 \mathrm{~min} / \mathrm{wk}$ & & \\
\hline & & & & - > $150 \mathrm{~min} / \mathrm{wk}$ & & \\
\hline \multirow{7}{*}{$\begin{array}{l}\text { Hjerkind } \\
\text { et al. } \\
\text { (2017) [37] }\end{array}$} & \multirow{7}{*}{$\begin{array}{l}\text { Norway (Nord- } \\
\text { Trøndelag } \\
\text { Health Study) }\end{array}$} & \multirow{7}{*}{$\begin{array}{l}N=38,413 \\
\text { with } \\
\text { information } \\
\text { on PA, } 47 \% \\
\text { males }\end{array}$} & 11 years & Self-reported LTPA & \multirow{7}{*}{$\begin{array}{l}\text { Incident diabetes (self- } \\
\text { reported; validated } \\
\text { through medical } \\
\text { record) }\end{array}$} & Risk of diabetes decreased \\
\hline & & & $\begin{array}{l}\text { Baseline: } \\
\text { 1984-1986 }\end{array}$ & - Low & & | Men: \\
\hline & & & & - Medium & & - Low: 1.0 (reference) \\
\hline & & & $\begin{array}{l}\text { Follow-up: } \\
\text { 1995-1997 }\end{array}$ & - High & & $\begin{array}{l}\text { - Medium: } 0.81[0.65,1.00] \mid \\
0.80[0.66,0.98]\end{array}$ \\
\hline & & & & & & $\begin{array}{l}\text { - High: } 0.76[0.61,0.95] \mid 0.65 \\
\quad[0.51,0.84] p=0.01 \mid p<0.01\end{array}$ \\
\hline & & & & & & $\begin{array}{l}\text { Gradual inverse association } \\
\text { between frequency, duration, } \\
\text { intensity and risk of incident } \\
\text { diabetes for males }\end{array}$ \\
\hline & & & & & & $\begin{array}{l}\text { Gradual inverse association } \\
\text { between frequency, intensity } \\
\text { and risk of incident diabetes } \\
\text { for females }\end{array}$ \\
\hline \multirow{4}{*}{$\begin{array}{l}\text { Ekelund } \\
\text { et al. } \\
\text { (2012) [42] }\end{array}$} & \multirow{4}{*}{$\begin{array}{l}8 \text { European } \\
\text { countries } \\
\text { (EPIC-InterAct } \\
\text { Study) }\end{array}$} & \multirow{2}{*}{$\begin{array}{l}N=11,669 \\
\text { men, } 15,695 \\
\text { women }\end{array}$} & \multirow[t]{2}{*}{$\begin{array}{l}\text { Median } 12.3 \\
\text { years }\end{array}$} & $\begin{array}{l}\text { Self-reported PA (OPA, } \\
\text { LTPA) }\end{array}$ & \multirow[t]{4}{*}{ Incident diabetes } & \multirow{4}{*}{$\begin{array}{l}\text { A one level difference in PA } \\
\text { (e.g. between inactive and } \\
\text { moderately inactive) was } \\
\text { associated with a } 13 \% \text { relative } \\
\text { reduction in risk of incident } \\
\text { diabetes in males ( } \mathrm{HR}[95 \% \mathrm{Cl}] \\
0.87[0.80,0.94] \text { ) and } 7 \% \text { risk }\end{array}$} \\
\hline & & & & - Inactive & & \\
\hline & & \multirow{2}{*}{$\begin{array}{l}N=15,934 \\
\text { subcohort } \\
6009 \text { men }\end{array}$} & $\begin{array}{l}\text { Baseline: } \\
1991\end{array}$ & - Moderately inactive & & \\
\hline & & & Follow-up: & - Moderately active & & \\
\hline
\end{tabular}


Table 3 Overview of longitudinal studies on the association between PA and the outcome of diabetes (Continued)

\begin{tabular}{|c|c|c|c|c|c|c|}
\hline Author & Country & Characteristics & $\begin{array}{l}\text { Follow-up } \\
\text { time }\end{array}$ & $\begin{array}{l}\text { Predictor variable: Physical } \\
\text { activity }\end{array}$ & Outcome of interest & Main results \\
\hline \multirow{11}{*}{$\begin{array}{l}\text { Jefferis } \\
\text { et al. } \\
\text { (2012) [38] }\end{array}$} & \multirow{11}{*}{ UK } & \multirow[t]{2}{*}{9925 women) } & \multirow[t]{2}{*}{2007} & \multirow[t]{2}{*}{ - Active } & & $\begin{array}{l}\text { reduction in females }(0.93 \\
[0.89,0.98])^{b}\end{array}$ \\
\hline & & & & & & $\begin{array}{l}\text { Increased risk of incident } \\
\text { diabetes associated with lower } \\
\text { levels of PA evident across BMI } \\
\text { strata in both sexes, with the } \\
\text { exception of obese women }\end{array}$ \\
\hline & & $N=3012$ & Median 7.1 & Self-reported PA & \multirow{9}{*}{$\begin{array}{l}\text { Incident type } 2 \text { diabetes } \\
\text { (self-report included } \\
\text { after validation through } \\
\text { medical record) }\end{array}$} & Risk of diabetes decreased with \\
\hline & & $\begin{array}{l}\text { men, } 08.3 \\
\text { years }\end{array}$ & & - None & & $\begin{array}{l}\text { PA: Dose-response assoctation: } \\
\text { HR [95\% Cl] }\end{array}$ \\
\hline & & & Baseline: & - Occasional & & - None: 1.0 (reference) \\
\hline & & & $\begin{array}{l}\text { 1990, 1998- } \\
2000 \\
\text { Follow-up: }\end{array}$ & - Light & & - Occasional: $0.54[0.31,0.96]$ \\
\hline & & & 2006 & - Moderate & & - Light: $0.34[0.18,0.65]$ \\
\hline & & & & - Moderately vigorous & & - Moderate: $0.33[0.17,0.65]$ \\
\hline & & & & - Vigorous & & $\begin{array}{l}\text { - moderately vigorous: } 0.32 \\
{[0.16,0.61]}\end{array}$ \\
\hline & & & & & & $\begin{array}{l}\text { - vigorous: } 0.26[0.13,0.53] \\
\quad p<0.01\end{array}$ \\
\hline & & & & & & $\begin{array}{l}\text { Taking up at least moderate } \\
\text { intensity PA also associated } \\
\text { with lower risk of diabetes. }\end{array}$ \\
\hline \multirow{6}{*}{$\begin{array}{l}\text { Koloverou } \\
\text { et al. } \\
\text { (2017) [39] }\end{array}$} & \multirow[t]{6}{*}{$\begin{array}{l}\text { Greece (Attica } \\
\text { Study) }\end{array}$} & \multirow[t]{6}{*}{$\begin{array}{l}N=1485,49 \% \\
\text { males }\end{array}$} & 10 years & $\begin{array}{l}\text { Self-reported PA (MET-min/ } \\
\text { wk) }\end{array}$ & \multirow{6}{*}{$\begin{array}{l}\text { Incident diabetes } \\
\text { (measured in biological } \\
\text { sample or self-reported) }\end{array}$} & \multirow{2}{*}{$\begin{array}{l}\text { Moderate intensity PA associated } \\
\text { with lower risk of incident } \\
\text { diabetes }^{\text {: }} \text { OR }[95 \% \mathrm{Cl}]\end{array}$} \\
\hline & & & \multirow{2}{*}{$\begin{array}{l}\text { Baseline: } \\
\text { 2001-2002 }\end{array}$} & - Very low $\leq 150$ & & \\
\hline & & & & - Low $=150-330$ & & - Very low: 1.0 (reference) \\
\hline & & & \multirow{3}{*}{$\begin{array}{l}\text { Follow-up: } \\
\text { 2011-2012 }\end{array}$} & - Moderate $=331-1484$ & & - Low: $0.77[0.41,1.49]$ \\
\hline & & & & - High $\geq 1484$ & & - Moderate: $0.47[0.24,0.93]$ \\
\hline & & & & & & - High: $1.04[0.59,1.82]$ \\
\hline \multirow{5}{*}{$\begin{array}{l}\text { Medina } \\
\text { et al. } \\
\text { (2018) [49] }\end{array}$} & \multirow{5}{*}{$\begin{array}{l}\text { Mexico } \\
\text { (Mexico City } \\
\text { Diabetes } \\
\text { Study) }\end{array}$} & \multirow{5}{*}{$\begin{array}{l}N=1883 \\
\text { median } 45 \\
\text { years (IQR } \\
\text { 39-52); } 42.7 \% \\
\text { males }\end{array}$} & $\begin{array}{l}\text { Median } 14.4 \\
\text { person years }\end{array}$ & \multirow{2}{*}{$\begin{array}{l}\text { Self-reported PA } \\
\text { (occupational, leisure, total } \\
\text { PA in MET-min/wk of MVPA) } \\
-1=<1\end{array}$} & \multirow{5}{*}{$\begin{array}{l}\text { Incident type } 2 \text { diabetes } \\
\text { (measured, self- } \\
\text { reported, taking } \\
\text { medication) }\end{array}$} & \multirow{2}{*}{$\begin{array}{l}\text { Participants with leisure } \\
\text { PA }<1 \text { MET-min/wk had } \\
\text { increased risk of incident } \\
\text { diabetes (HR } 1.45 \text { [ } 95 \% \text { Cl: 1.10, } \\
\text { 1.92]) as compared to reference } \\
\text { group }(\geq 1200 \text { MET-min/wk } \\
\text { of MVPA; } p=0.008)^{e}\end{array}$} \\
\hline & & & $\begin{array}{l}\text { Baseline: } \\
\text { 1989-1990 }\end{array}$ & & & \\
\hline & & & \multirow{3}{*}{$\begin{array}{l}\text { Follow-up: } \\
\text { 1993-1994, } \\
\text { 1997-1998, } \\
\text { 2008-2009 }\end{array}$} & $-2=1-599.9$ & & \multirow{3}{*}{$\begin{array}{l}\text { No association between } \\
\text { occupational and total PA and } \\
\text { diabetes risk. }\end{array}$} \\
\hline & & & & $-3=600-1199.9$ & & \\
\hline & & & & $-4=\geq 1200$ & & \\
\hline \multirow{6}{*}{$\begin{array}{l}\text { Mehlig } \\
\text { et al. } \\
\text { (2014) [43] }\end{array}$} & \multirow[t]{6}{*}{ Sweden } & \multirow{6}{*}{$\begin{array}{l}N=1448 \\
\text { women, } 38- \\
60 \text { years }\end{array}$} & 34 years & Self-reported LTPA & \multirow[t]{6}{*}{ Incident diabetes } & LTPA is associated with an \\
\hline & & & \multirow{2}{*}{$\begin{array}{l}\text { Baseline: } \\
\text { 1968-1969 }\end{array}$} & - Almost inactive: low LTPA & & diabetes: HR $[95 \% \mathrm{CI}]^{f}$ \\
\hline & & & & - Some PA at least $4 \mathrm{~h} / \mathrm{wk}$ & & $\begin{array}{l}\text { - Non-obese, active: } 1.0 \\
\text { (reference) }\end{array}$ \\
\hline & & & \multirow{3}{*}{$\begin{array}{l}\text { Follow-up: } \\
\text { 1974-1975, } \\
\text { 1980-1981, } \\
\text { 1992-1993, } \\
2000-2001 \\
2000\end{array}$} & - Regular exercise & & $\begin{array}{l}\text { - Non-obese, inactive: } 1.79 \\
{[1.15,2.79]}\end{array}$ \\
\hline & & & & $\begin{array}{l}\text { - Regular training and com- } \\
\text { petitive sports }\end{array}$ & & $\begin{array}{l}\text { - Obese, active: } 2.43 \\
{[1.44,4.09]}\end{array}$ \\
\hline & & & & & & $\begin{array}{l}\text { - Obese, inactive: } 11.7 \\
{[6.28,21.8]}\end{array}$ \\
\hline $\begin{array}{l}\text { Shi et al. } \\
\text { (2013) [44] }\end{array}$ & China & $\begin{array}{l}N=51,464 \\
\text { men, } 54.1 \pm \\
9.3 \text { years }\end{array}$ & $\begin{array}{l}\text { Median } 5.4 \\
\text { years }\end{array}$ & $\begin{array}{l}\text { Self-reported PA MET level } \\
\text { (in quintiles) }\end{array}$ & $\begin{array}{l}\text { Incident diabetes (self- } \\
\text { reported) }\end{array}$ & $\begin{array}{l}\text { Total PA is associated with a } \\
\text { reduced risk in incident diabetes: } \\
\text { HR }\left[95 \% \mathrm{CI} \text { for MET level }{ }^{9}\right.\end{array}$ \\
\hline
\end{tabular}


Table 3 Overview of longitudinal studies on the association between PA and the outcome of diabetes (Continued)

\begin{tabular}{|c|c|c|c|c|c|c|}
\hline Author & Country & Characteristics & $\begin{array}{l}\text { Follow-up } \\
\text { time }\end{array}$ & $\begin{array}{l}\text { Predictor variable: Physical } \\
\text { activity }\end{array}$ & Outcome of interest & Main results \\
\hline \multirow{12}{*}{$\begin{array}{l}\text { Williams \& } \\
\text { Thompson } \\
\text { (2012) [50] }\end{array}$} & \multirow{12}{*}{ USA } & \multirow{12}{*}{$N=48,116$} & \multirow{2}{*}{$\begin{array}{l}\text { Baseline: } \\
\text { 2002-2006 }\end{array}$} & $-\mathrm{Q} 1<4.3$ & & - Q1: 1.0 (reference) \\
\hline & & & & - Q2 4.3-6.5 & & - Q2: $0.84[0.72,0.99]$ \\
\hline & & & \multirow{3}{*}{$\begin{array}{l}\text { Follow-up: } \\
\text { 2004-2008, } \\
\text { 2008-2011 }\end{array}$} & - Q3 6.5-8.9 & & - Q3: $0.72[0.61,0.85]$ \\
\hline & & & & - Q4 8.9-12.1 & & - Q4: $0.66[0.55,0.78]$ \\
\hline & & & & $-\mathrm{Q} 5 \geq 12.1$ & & - Q5: $0.65[0.54,0.77]$ \\
\hline & & & Median 6.2 & Self-reported PA MET-h/d & \multirow[t]{7}{*}{ Incident diabetes } & Greater MET-h/d is associated \\
\hline & & & & - Light & & diabetes: HR $[95 \% \mathrm{Cl}]^{\mathrm{h}}$ \\
\hline & & & \multirow{2}{*}{$\begin{array}{l}\text { Baseline: } \\
\text { 1998-1999 }\end{array}$} & - Moderate & & - Running: $0.879[0.83,0.929]$ \\
\hline & & & & - Vigorous & & - Walking: $0.877[0.82,0.93]$ \\
\hline & & & \multirow[t]{3}{*}{$\begin{array}{l}\text { Follow-up: } \\
2006\end{array}$} & & & $\begin{array}{l}\text { - Other vigorous: } 0.98 \text { [0.95, } \\
1.007]\end{array}$ \\
\hline & & & & & & $\begin{array}{l}\text { - Other moderate: } 0.969 \text { [0.908, } \\
\text { 1.02] }\end{array}$ \\
\hline & & & & & & - Other light: $0.99[0.736,1.12]$ \\
\hline
\end{tabular}

Abbreviation: BP - $C l$ confidence interval, $d$ day, $h$ hour, $H R$ hazard ratio, IQR interquartile range, $L T P A$ leisure time physical activity, MET metabolic equivalent, min minutes, MVPA moderate to vigorous physical activity, $N$ number of participants, OPA occupational physical activity, $O R$ odds ratio, $P A$ physical activity, RR relative risk, wk week

a: Model adjusted for age; education, alcohol frequency in the past 2 weeks, smoking, blood pressure medication use, prevalent cardiovascular disease, BMI, PA summary score; b Model adjusted for study center, education, smoking status, alcohol consumption, energy intake, BMl; ${ }^{c}$ Model adjusted for age $\&$ region; ${ }^{d}$ Model adjusted for age, sex, family history of diabetes, hypertension, hypercholesterolemia, smoking status, education, physical activity, waist circumference, adherence to the Mediterranean diet, fasting glucose, triglycerides; ${ }^{\mathrm{e}}$ Model adjusted for sex, age, education levels, marital status, current smoking, alcohol intake, total energy intake, parent history of diabetes, sleeping hours, leisure/working MET-min/wk; ${ }^{\mathrm{f}}$ Model adjusted for baseline covariates age, education, smoking, consumption of alcohol, triglycerides, hypertension, parental history of diabetes (diabetes only); ${ }^{9}$ Model adjusted for age at interview, energy intake, smoking, alcohol consumption, education level, occupation, income level, hypertension, family history of diabetes; ${ }^{\mathrm{h}}$ Model adjusted for baseline age (age, age2), sex, race, education, smoking, intakes of red meat, fruit, alcohol, preexisting CHD at baseline

reduction of incident hypertension by $6 \%$ for each 10 metabolic equivalent of task hours per week increment of leisure time PA [58]. However, another meta-analysis detected an inverse association for recreational PA and incident hypertension but not for occupational PA [59]. Of note, the causes of hypertension are multifactorial and the way they interact and ultimately contribute to the development of hypertension is unclear. Thus, potential mechanisms for prevention of hypertension through PA also remain unclear.

Some studies included in this review also reported findings stratified by sex and body weight. For example, one study observed a gradual inverse association between frequency, duration and intensity of PA and risk of incident diabetes in males, but only a gradual inverse association between frequency and intensity of PA and risk of incident diabetes in females [37]. Additionally, another study reported that, while overall PA irrespective of body weight was not associated with the outcome of incident hypertension, obese males with high PA had a significantly lower risk of hypertension than obese males with low PA [47].

The quality of included studies was independently assessed by two reviewers and was rated as moderate to good, with scores ranging between 16 and 22 (total range: 0-22). This is not surprising since we only included studies published in or after 2012 that may already have followed quality guidelines on reporting findings of observational studies such as STROBE [23]. The potential risk of bias was rated moderate to poor and there were several concerns that warrant brief discussion: 1) All studies included in this review assessed PA through self-reported questionnaires which may be prone to recall bias. However, given the large sample sizes and since the baseline measurements of PA of many studies took place several years or even decades ago, objective measurement of PA might not have been feasible. There is good reason to believe that more longitudinal studies using novel objective techniques such as accelerometry (e.g. $[60,61]$ ) will become available in the near future. 2) The studies differed regarding the assessment of the outcomes of interest, e.g. some studies objectively measured blood glucose levels or blood pressure (e.g. [47, 49]) whereas others relied on selfreported information by the study participants and/or medical chart review (e.g. [40, 48]). 3) The studies differed in terms of adjustment for potential confounders and mediators which makes a comparison of findings between studies difficult. 4) Five studies were conducted only among males [30, 31, 38, 40, 44] and five studies were conducted only among females [28, 41, 43, 46, 48].

We did not investigate potential mechanisms underlying the associations between PA and incident obesity, 
Table 4 Overview of longitudinal studies on the association between PA and the outcome of hypertension

\begin{tabular}{|c|c|c|c|c|c|c|}
\hline Author & Country & Characteristics & $\begin{array}{l}\text { Follow-up } \\
\text { time }\end{array}$ & $\begin{array}{l}\text { Predictor variable: } \\
\text { Physical activity }\end{array}$ & $\begin{array}{l}\text { Outcome of } \\
\text { interest }\end{array}$ & Main results \\
\hline \multirow{6}{*}{$\begin{array}{l}\text { Cohen } \\
\text { et al. } \\
(2012)[48]\end{array}$} & \multirow{6}{*}{$\begin{array}{l}\text { USA (Nurses' } \\
\text { Health Study I } \\
\text { cohort) }\end{array}$} & \multirow{6}{*}{$\begin{array}{l}N=78,590 \\
\text { women; } 49 \\
\text { years (IQR 44- } \\
56)\end{array}$} & 20 years & Self-reported PA & \multirow{6}{*}{$\begin{array}{l}\text { Incident } \\
\text { hypertension } \\
\text { (self-reported; } \\
\text { validated in NHS } \\
\text { I cohort) }\end{array}$} & \multirow{2}{*}{$\begin{array}{l}\text { Association between PA and incident } \\
\text { hypertension varies by age ( } \mathrm{p} \text {-value for } \\
\text { interaction }<0.001) \text {. }\end{array}$} \\
\hline & & & $\begin{array}{l}\text { Baseline: } \\
1984\end{array}$ & \multirow{5}{*}{$\begin{array}{l}\text { METs/wk for vigorous } \\
\text { exercise in quintiles } \\
\text { (Q1-5) }\end{array}$} & & \\
\hline & & & \multirow[t]{4}{*}{$\begin{array}{l}\text { Follow-up: } \\
2004\end{array}$} & & & $\begin{array}{l}\text { HR }[95 \% \mathrm{Cl} \text { lowest for PA Q5 as } \\
\text { compared to Q1. }\end{array}$ \\
\hline & & & & & & $\begin{array}{l}\text { - Age } \geq 50: \text { Q1 } 1.0 \text { (reference); Q2 } 1.00 \\
\text { [0.91, 1.11]; Q3 } 1.03[0.94,1.14] ; \text { Q4 } 1.01 \\
{[0.91,1.12] ; \text { Q5 } 0.87[0.78,0.97]}\end{array}$ \\
\hline & & & & & & $\begin{array}{l}\text { - Age 51-60: Q1 } 1.0 \text { (reference); Q2 } 0.94 \\
\text { [0.88, 1.00]; Q3 } 0.94[0.88,1.00] ; \text { Q4 } 0.91 \\
{[0.85,0.97] ; \text { Q5 } 0.86[0.80,0.92]}\end{array}$ \\
\hline & & & & & & $\begin{array}{l}\text { - Age } \geq 61: \text { Q1 } 1.0 \text { (reference); Q2 } 1.03 \\
\quad[0.97,1.09] ; \text { Q3 } 0.98[0.93,1.04] ; \text { Q4 } 0.99 \\
{[0.93,1.05] ; \text { Q5 } 0.95[0.90,1.01]}\end{array}$ \\
\hline \multirow{5}{*}{$\begin{array}{l}\text { Lu et al. } \\
\text { (2015) [45] }\end{array}$} & \multirow[t]{5}{*}{ China } & \multirow{5}{*}{$\begin{array}{l}N=1009 \\
35.48 \pm 0.19 \\
\text { years, } 63.4 \% \\
\text { males }\end{array}$} & \multirow{2}{*}{$\begin{array}{l}\text { Median } 4.7 \\
\text { years }\end{array}$} & Self-reported PA & \multirow{5}{*}{$\begin{array}{l}\text { Incident } \\
\text { hypertension }\end{array}$} & No significant association between PA \\
\hline & & & & - Frequent & & and risk of hypertension : HR [95\% Cl] \\
\hline & & & $\begin{array}{l}\text { Base line: } \\
2004\end{array}$ & - Occasional & & - Occasional: $0.74[0.40,1.39]$ \\
\hline & & & \multirow{2}{*}{$\begin{array}{l}\text { Follow-up: } \\
2012\end{array}$} & \multirow[t]{2}{*}{ - Everyday } & & - Frequent: $0.96[0.51,1.83]$ \\
\hline & & & & & & - Everyday: 1.0 (reference) \\
\hline \multirow{6}{*}{$\begin{array}{l}\text { Medina } \\
\text { et al. } \\
\text { (2018) [49] }\end{array}$} & \multirow{6}{*}{$\begin{array}{l}\text { Mexico (Mexico } \\
\text { City Diabetes } \\
\text { Study) }\end{array}$} & \multirow{6}{*}{$\begin{array}{l}N=1541 \\
\text { median } 45 \\
\text { (IQR 39-52) } \\
\text { years; } 45.1 \% \\
\text { males }\end{array}$} & $\begin{array}{l}\text { Median } 11.8 \\
\text { years }\end{array}$ & \multirow{3}{*}{$\begin{array}{l}\text { Self-reported PA } \\
\text { (occupational, leisure, } \\
\text { total activity in MET- } \\
\text { min/wk of MVPA) } \\
-1=<1\end{array}$} & \multirow{6}{*}{$\begin{array}{l}\text { Incident } \\
\text { hypertension } \\
\text { (measured by } \\
\text { study team) }\end{array}$} & \multirow{3}{*}{$\begin{array}{l}\text { Participants with }<1 \text { MET-min/wk of leis- } \\
\text { ure (HR } 1.37[95 \% \mathrm{Cl} 1.07,1.75], p=0.015) \\
\text { or occupational MVPA (HR 1.52 [1.17, } \\
\text { 1.97], } p=0.001 \text { ) had increased risk of } \\
\text { hypertension as compared to reference } \\
\text { group }(\geq 1200 \mathrm{MET}-\mathrm{min} / \mathrm{wk})^{\mathrm{b}}\end{array}$} \\
\hline & & & $\begin{array}{l}\text { Baseline: } \\
\text { 1989-1990 }\end{array}$ & & & \\
\hline & & & & & & \\
\hline & & & \multirow{3}{*}{$\begin{array}{l}\text { Follow-up: } \\
\text { 1993-1994, } \\
\text { 1997-1998, } \\
\text { 2008-2009 }\end{array}$} & $-2=1-599.9$ & & \multirow{3}{*}{$\begin{array}{l}\text { No association was observed between } \\
\text { total PA and hypertension. }\end{array}$} \\
\hline & & & & $-3=600-1199.9$ & & \\
\hline & & & & $-4=\geq 1200$ & & \\
\hline \multirow[t]{11}{*}{$\begin{array}{l}\text { Pavey et al. } \\
\text { (2013) [46] }\end{array}$} & \multirow{11}{*}{$\begin{array}{l}\text { Australia } \\
\text { (Australian } \\
\text { Longitudinal } \\
\text { Study on } \\
\text { Women's Health) }\end{array}$} & \multirow{11}{*}{$\begin{array}{l}N=11,285 \\
\text { women, mean } \\
\text { age } 49.5 \text { years }\end{array}$} & $\begin{array}{l}\text { Baseline: } \\
1998\end{array}$ & $\begin{array}{l}\text { Self-reported PA } \\
\text { (MET-min/wk) }\end{array}$ & \multirow{11}{*}{$\begin{array}{l}\text { Occurrence of } \\
\text { hypertension } \\
\text { (self-reported) }\end{array}$} & \multirow{3}{*}{$\begin{array}{l}\text { OR }[95 \% \mathrm{CI}] \text { for hypertension declined } \\
\text { with increasing PA volume; decline } \\
\text { slightly greater in MVPA than MPA group } \\
\text { MPA }^{c} \mid \text { MVPA }^{c}\end{array}$} \\
\hline & & & \multirow{10}{*}{$\begin{array}{l}\text { Follow-up: } \\
\text { 2001, 2004, } \\
2007,2010\end{array}$} & - None & & \\
\hline & & & & $->0-<250$ & & \\
\hline & & & & $-250-<500$ & & - None: 1.0 (reference) \\
\hline & & & & $-500-<1000$ & & $\begin{array}{l}->0-<250: 0.92[0.83,1.02] \mid 0.87[0.63 \\
1.04]\end{array}$ \\
\hline & & & & $-1000-<1500$ & & $-250-<500: 0.90[0.81,1.00]$ | N.A. \\
\hline & & & & $-1500-<2000$ & & $\begin{array}{l}-500-<1000: 0.82[0.75,0.91] \mid 0.73[0.62 \\
0.86]\end{array}$ \\
\hline & & & & $->2000$ & & $\begin{array}{l}-1000-<1500: 0.74[0.66,0.82] \mid 0.65 \\
{[0.55,0.76]}\end{array}$ \\
\hline & & & & - Inactive & & $\begin{array}{l}-1500-<2000: 0.78[0.68,0.90] \mid 0.63 \\
\quad[0.54,0.74]\end{array}$ \\
\hline & & & & - Moderate (MPA) & & - > 2000: $0.80[0.70,0.93] \mid 0.56[0.49,0.64]$ \\
\hline & & & & $\begin{array}{l}\text { - Moderate and } \\
\text { vigorous activity } \\
\text { (MVPA) }\end{array}$ & & \\
\hline \multirow{4}{*}{$\begin{array}{l}\text { Stenehjem } \\
\text { et al. } \\
\text { (2018) [47] }\end{array}$} & Norway (Nord- & $N=21,892$ & 11 years & Self-reported LTPA & Incident & Risk of hypertension not associated with \\
\hline & $\begin{array}{l}\text { Irøndelag Health } \\
\text { Study) }\end{array}$ & & Baseline: & Total score & $\begin{array}{l}\text { nypertension } \\
\text { (measured by }\end{array}$ & \\
\hline & & & & - Low & study team) & Women | Men \\
\hline & & & Follow-up: & - Medium & & - Low: 1.0 (reference) \\
\hline
\end{tabular}


Table 4 Overview of longitudinal studies on the association between PA and the outcome of hypertension (Continued)

\begin{tabular}{|c|c|c|c|c|c|c|}
\hline Author & Country & Characteristics & $\begin{array}{l}\text { Follow-up } \\
\text { time }\end{array}$ & $\begin{array}{l}\text { Predictor variable: } \\
\text { Physical activity }\end{array}$ & $\begin{array}{l}\text { Outcome of } \\
\text { interest }\end{array}$ & Main results \\
\hline & & & 1995-1997 & High & & $\begin{array}{l}\text { - Medium: } 0.98[0.92,1.05] \mid 0.96[0.90 \text {, } \\
\text { 1.03] }\end{array}$ \\
\hline & & & & Frequency (per wk) & & - High: $0.96[0.90,1.01]$ | $0.97[0.90,1.03]$ \\
\hline & & & & - None & & $0.138 \mid p=0.2 / 6$ \\
\hline & & & & $-<1$ & & Frequency of PA associated with reduced \\
\hline & & & & -1 & & $\begin{array}{l}\text { risk of hypertension only in males ( } 24 / \mathrm{Wk} \text { : } \\
\text { RR } 0.87[0.78,0.98]) \text {. }\end{array}$ \\
\hline & & & & $-2-3$ & & Obese males with high PA have lower \\
\hline & & & & $-\geq 4$ & & $\begin{array}{l}\text { than obese males with low PA (RR } 1.50 \\
[1,27,1.77]) \text {. }\end{array}$ \\
\hline & & & & Intensity & & Obese females with low PA have \\
\hline & & & & - None & & $\begin{array}{l}\text { increased risk of hypertension (RR } 1.55 \\
[1.35,1.77]) \text {. }\end{array}$ \\
\hline & & & & - Low & & \\
\hline & & & & - Medium/high & & \\
\hline \multirow{7}{*}{$\begin{array}{l}\text { Williams \& } \\
\text { Thompson } \\
\text { (2012) [50] }\end{array}$} & \multirow[t]{7}{*}{ USA } & \multirow[t]{7}{*}{$N=43,893$} & Median 6.2 & Self-reported PA & \multirow{7}{*}{$\begin{array}{l}\text { Incident } \\
\text { hypertension }\end{array}$} & \multirow{2}{*}{$\begin{array}{l}\text { Greater MET-h/d is associated with lower } \\
\text { risk of incident hypertension: HR [95\% Cl] }\end{array}$} \\
\hline & & & years & MET-h/d & & \\
\hline & & & \multirow{2}{*}{$\begin{array}{l}\text { Baseline: } \\
\text { 1998-1999 }\end{array}$} & - Light & & - Running: $0.958[0.94,0.97]$ \\
\hline & & & & - Moderate & & - Walking: 0.928 [0.899, 0.957] \\
\hline & & & \multirow{3}{*}{$\begin{array}{l}\text { Follow-up: } \\
2006\end{array}$} & \multirow[t]{3}{*}{ - Vigorous } & & - Other vigorous: 0.98 [0.97, 0.99] \\
\hline & & & & & & - Other moderate: 0.997 [0.976, 1.018] \\
\hline & & & & & & - Other light: $0.886[0.739,1.006]$ \\
\hline
\end{tabular}

Abbreviation: $C l$ confidence interval, $d$ day, $h$ hour, HR hazard ration, IQR interquartile range, LTPA leisure time physical activity, MET metabolic equivalent, min minutes, MPA moderate physical activity, MVPA moderate to vigorous physical activity, $N$ number of participants, N.A. not available, $P$ p-value, $P A$ physical activity, $R R$ relative risk, SE standard error, wk week

a: Model adjusted for age, gender and follow-up time; ${ }^{b}$ Model adjusted for sex*time, age, education levels, marital status, current smoking, alcohol intake*time, total energy intake, sleeping hours, leisure/ working METs/min/wk; ${ }^{c}$ Model adjusted for sociodemographic (age, education, marital status, area of residence), behavioral (smoking, alcohol, and sitting), chronic conditions covariates; ${ }^{d}$ Model adjusted for age, marital status, education, smoking, alcohol frequency last 2 weeks, BMI, PA summary score; ${ }^{e}$ Model adjusted for baseline age (age, age2), sex, race, education, smoking, intakes of red meat, fruit, alcohol, preexisting CHD at baseline

diabetes and CHD. However, it has previously been postulated that there are acute and chronic effects of PA on insulin resistance, which may account for improvements in insulin action and decreased blood glucose levels as a response to engagement in PA [62]. Additionally, PA impacts energy balance by increasing total energy expenditure, which in turn causes an energy deficit and may lead to lower body weight [63]. Stimulating responses in adipose and body tissues by PA may also influence total energy balance and body composition [63]. Furthermore, studies suggest that regular PA increases capillarization and may reverse endothelial alterations, which is a major risk factor for CHD [64].

The strengths of this review are the rigorous search and selection strategy following published guidelines and conducted by two reviewers. Also, both quality and potential risk of bias were assessed by two authors independently. We deliberately focused on studies published after January of 2012 in order to provide an update of a systematic review previously published by our group [20]. In addition, even though unintentional, our review included studies from various countries such as Sweden, Norway, UK, Greece, Mexico, China, Australia, Italy, US, the Netherlands, Germany, France, Spain and Denmark which may add to the generalizability of our observations. The major limitations of this review pertain to the relatively small number of included studies. This may be due to the fact that we only included studies with large sample size $(N \geq 500)$ and relatively long follow-up time of $\geq 5$ years. However, we believe that these criteria ensure validity of our conclusions and a higher probability of generalizability of the study findings. In addition, a large body of research on PA and overweight/obesity published after 2012 focused on change in BMI or body weight over time. We opted to not include these studies in our review as we chose our outcome of interest to be incident obesity, and information on change in BMI or body weight over time is thus not sufficient. For instance, a person could be underweight at baseline and an increase in BMI or weight might actually reflect progression to a healthier body constitution. At the same time, we also acknowledge that particularly obesity and 
hypertension are conditions for which individuals can take action to improve, i.e., a person develops incident obesity but may be able to decrease body weight in order to progress back to overweight or normal weight. Furthermore, our search terms may have been too narrow or not comprehensive enough and there may be published studies that we were not able to identify. However, in order to compensate for this potential shortcoming, we also manually searched bibliographies of included studies. Finally, the studies differed with regard to the depth of investigating PA variables. As such, PA was only one of many predictors in some studies (e.g. [36, 42]) and thus only one finding related to PA and the outcome of interest was reported. Whereas in other studies, the association between various PA parameters (e.g. type, intensity, frequency, duration) and the outcome of interest was examined (e.g. $[30,47])$.

More research to untangle the association and potential underlying mechanisms between PA and the outcome of incident overweight, $\mathrm{CHD}$, diabetes and hypertension is needed, preferably using prospective cohort studies with large sample sizes, long follow-up and objective measurement of both predictor variable (i.e. PA) and the outcomes of interest. In addition, metaanalytic approaches to address research questions pertaining the association between PA and various health outcomes are warranted.

\section{Conclusion}

Overall, this systematic review replicates, updates, and extends the growing body of research on the associations between PA and incident obesity, CHD and diabetes. No clear association between PA and reduced risk for hypertension was detected. This review emphasises the contribution of PA in the prevention of various chronic diseases. Reducing the risk of new onset of NCDs and thereby reducing the economic burden on health systems is of high importance to societies worldwide. Regional and global action plans and preventive strategies (e.g. [65]) should highlight the beneficial impact of regular PA and support national governments in the implementation of concrete actions towards achieving a higher engagement in PA among individuals across all ages.

\section{Abbreviations}

BMI: Body mass index; CHD: Coronary heart disease; NCDs: Noncommunicable diseases; PA: Physical activity; PRISMA: Preferred Reporting Items for Systematic Reviews and Meta-Analysis

\section{Authors' contributions}

LC \& JKR conducted the literature search, and extracted and interpreted data. LC wrote the first draft of the manuscript. JKR, CN and AW revised the manuscript. All authors read and approved the final manuscript.

\section{Funding}

$\mathrm{CN}$ received funding by the German Academic Exchange Service (DAAD) for a visiting professorship at KIT.

\section{Availability of data and materials}

All data generated or analyzed during this study are included in this published article.

Ethics approval and consent to participate

Not applicable.

Consent for publication

Not applicable.

\section{Competing interests}

All authors declare no conflicts of interest pertaining to this manuscript.

\section{Author details}

${ }^{1}$ Institute of Sports and Sports Science, Karlsruhe Institute of Technology, Karlsruhe, Germany. ${ }^{2}$ Translational Neuroscience and Aging Laboratory, Mayo Clinic, Scottsdale, AZ, USA. ${ }^{3}$ Present address: Institute of Sports Science, University of Bern, Bern, Switzerland.

Received: 29 May 2019 Accepted: 15 April 2020

Published online: 19 May 2020

\section{References}

1. World Health Organization. Global status report on noncommunicable diseases 2014. Geneva: World Health Organization; 2014.

2. The Lancet. GBD 2017: a fragile world. Lancet. 2018;392:1683. https://doi. org/10.1016/S0140-6736(18)32858-7.

3. Foreman KJ, Marquez N, Dolgert A, Fukutaki K, Fullman N, McGaughey M, et al. Forecasting life expectancy, years of life lost, and all-cause and causespecific mortality for 250 causes of death: reference and alternative scenarios for 2016-40 for 195 countries and territories. Lancet. 2018;392: 2052-90. https://doi.org/10.1016/S0140-6736(18)31694-5.

4. Arts J, Fernandez ML, Lofgren IE. Coronary heart disease risk factors in college students. Adv Nutr. 2014;5:177-87. https://doi.org/10.3945/an.113.005447.

5. Forouzanfar MH, Afshin A, Alexander LT, Anderson HR, Bhutta ZA, Biryukov $S$, et al. Global, regional, and national comparative risk assessment of 79 behavioural, environmental and occupational, and metabolic risks or clusters of risks, 1990-2015: a systematic analysis for the global burden of disease study 2015. Lancet. 2016;388:1659-724. https://doi.org/10.1016/ S0140-6736(16)31679-8

6. Madjd A, Taylor MA, Shafiei Neek L, Delavari A, Malekzadeh R, Macdonald IA Farshchi HR. Effect of weekly physical activity frequency on weight loss in healthy overweight and obese women attending a weight loss program: a randomized controlled trial. Am J Clin Nutr. 2016;104:1202-8. https://doi. org/10.3945/ajcn.116.136408

7. Hambrecht R, Wolf A, Gielen S, Linke A, Hofer J, Erbs S, et al. Effect of exercise on coronary endothelial function in patients with coronary artery disease. N Engl J Med. 2000;342:454-60. https://doi.org/10.1056/ NEJM200002173420702

8. Colditz GA, Philpott SE, Hankinson SE. The impact of the Nurses' health study on population health: prevention, translation, and control. Am J Public Health. 2016;106:1540-5. https://doi.org/10.2105/AJPH.2016.303343

9. Johannsen NM, Swift DL, Lavie CJ, Earnest CP, Blair SN, Church TS. Categorical analysis of the impact of aerobic and resistance exercise training, alone and in combination, on cardiorespiratory fitness levels in patients with type 2 diabetes: results from the HART-D study. Diabetes Care. 2013;36:3305-12. https://doi.org/10.2337/dc12-2194

10. Church TS, Blair SN, Cocreham S, Johannsen N, Johnson W, Kramer K, et al. Effects of aerobic and resistance training on hemoglobin A1c levels in patients with type 2 diabetes: a randomized controlled trial. JAMA. 2010; 304:2253-62. https://doi.org/10.1001/jama.2010.1710

\section{Acknowledgements}

Not applicable. 
11. Stewart KJ, Bacher AC, Turner KL, Fleg $J$, Hees PS, Shapiro EP, et al. Effect of exercise on blood pressure in older persons: a randomized controlled trial. Arch Intern Med. 2005;165:756-62. https://doi.org/10. 1001/archinte.165.7.756

12. Nelson L, Esler M, Jennings $G$, Korner P. Effect of changing levels of physical activity on blood-pressure and haemodynamics in essential hypertension. Lancet. 1986;328:473-6. https://doi.org/10.1016/S0140-6736(86)90354-5 .

13. Avery MD, Walker AJ. Acute effect of exercise on blood glucose and insulin levels in women with gestational diabetes. J Matern Fetal Neonatal Med. 2001;10:52-8. https://doi.org/10.1080/jmf.10.1.52.58-4

14. Fritz T, Rosenqvist U. Walking for exercise? Immediate effect on blood glucose levels in type 2 diabetes. Scand J Prim Health Care. 2001;19:31-3. https://doi.org/10.1080/pri.19.1.31.33 .

15. Leon AS. Leisure-time physical activity levels and risk of coronary heart disease and death. JAMA. 1987;258:2388. https://doi.org/10.1001/jama.1987. 03400170074026 .

16. Sesso HD, Paffenbarger RS, Lee IM. Physical activity and coronary heart disease in men: the Harvard alumni health study. Circulation. 2000;102:975-80.

17. Paffenbarger RS, Laughlin ME, Gima AS, Black RA. Work activity of longshoremen as related to death from coronary heart disease and stroke. N Engl J Med. 1970;282:1109-14. https://doi.org/10.1056/ NEJM197005142822001

18. Sherman SE, D'Agostino RB, Cobb JL, Kannel WB. Does exercise reduce mortality rates in the elderly? Experience from the Framingham heart study. Am Heart J. 1994;128:965-72.

19. Kannel WB, Belanger A, D'Agostino R, Israel I. Physical activity and physical demand on the job and risk of cardiovascular disease and death: the Framingham study. Am Heart J. 1986;112:820-5. https://doi.org/10.1016/ 0002-8703(86)90480-1.

20. Reiner M, Niermann C, Jekauc D, Woll A. Long-term health benefits of physical activity-a systematic review of longitudinal studies. BMC Public Health. 2013;13:813. https://doi.org/10.1186/1471-2458-13-813 .

21. World Health Organization. A global brief on Hypertension. 2013. http:// apps.who.int/iris/bitstream/handle/10665/79059/WHO_DCO_WHD_2013.2 eng.pdf;jsessionid=9E19A6932A6A21A76238A0826D725EC9? sequence $=1$. Accessed 9 Jan 2019.

22. Moher D, Liberati A, Tetzlaff J, Altman DG. Preferred reporting items for systematic reviews and meta-analyses: the PRISMA statement. PLOS Med. 2009;6:e1000097. https://doi.org/10.1371/journal.pmed.1000097.

23. von Elm E, Altman DG, Egger M, Pocock SJ, Gøtzsche PC, Vandenbroucke JP. The strengthening the reporting of observational studies in epidemiology (STROBE) statement: guidelines for reporting observational studies. Lancet. 2007;370:1453-7. https://doi.org/10.1016/S0140-6736(07)61602-X.

24. Tool to Assess Risk of Bias in Cohort Studies. http://methods.cochrane.org/ sites/methods.cochrane.org.bias/files/public/uploads/Tool\%20to\%20Assess\%2 ORisk\%20of\%20Bias\%20in\%20Cohort\%20Studies.pdf. Accessed 15 Apr 2019.

25. Bell JA, Hamer M, Batty GD, Singh-Manoux A, Sabia S, Kivimaki M. Combined effect of physical activity and leisure time sitting on long-term risk of incident obesity and metabolic risk factor clustering. Diabetologia. 2014;57:2048-56. https://doi.org/10.1007/s00125-014-3323-8 .

26. Montgomerie AM, Chittleborough CR, Taylor AW. Physical inactivity and incidence of obesity among south Australian adults. PLoS One. 2014;9: e112693. https://doi.org/10.1371/journal.pone.0112693

27. Pavey TG, Peeters GMEE, Gomersall SR, Brown WJ. Long-term effects of physical activity level on changes in healthy body mass index over 12 years in young adult women. Mayo Clin Proc. 2016;91:735-44. https://doi.org/10. 1016/j.mayocp.2016.03.008

28. Chomistek AK, Henschel B, Eliassen AH, Mukamal KJ, Rimm EB. Frequency, type, and volume of leisure-time physical activity and risk of coronary heart disease in young women. Circulation. 2016;134:290-9. https://doi.org/10. 1161/CIRCULATIONAHA.116.021516

29. Delaney JAC, Jensky NE, Criqui MH, Whitt-Glover MC, Lima JAC, Allison MA. The association between physical activity and both incident coronary artery calcification and ankle brachial index progression: the multi-ethnic study of atherosclerosis. Atherosclerosis. 2013;230:278-83.

30. Ferrario MM, Roncaioli M, Veronesi G, Holtermann A, Clays E, Borchini R, et al. Differing associations for sport versus occupational physical activity and cardiovascular risk. Heart. 2018;104:1165-72. https://doi.org/10.1136/ heartjnl-2017-312594.

31. Jefferis BJ, Whincup PH, Lennon LT, Papacosta O, Goya Wannamethee S. Physical activity in older men: Longitudinal associations with inflammatory and hemostatic biomarkers, N-terminal pro-brain natriuretic peptide, and onset of coronary heart disease and mortality. J Am Geriatr Soc. 2014;62:599-606.

32. Koolhaas CM, Dhana K, Golubic R, Schoufour JD, Hofman A, Van Rooij FJA, Franco OH. Physical Activity Types and Coronary Heart Disease Risk in Middle-Aged and Elderly Persons: The Rotterdam Study. Am J Epidemiol. 2016:183:729-38.

33. Petersen CB, Gronbaek M, Helge JW, Thygesen LC, Schnohr P, Tolstrup JS. Changes in physical activity in leisure time and the risk of myocardial infarction, ischemic heart disease, and all-cause mortality. Eur J Epidemiol. 2012;27:91-9.

34. Soares-Miranda L, Siscovick DS, Psaty BM, Longstreth WT, Mozaffarian D. Physical activity and risk of coronary heart disease and stroke in older adults. Circulation. 2016;133:147-55.

35. Tikkanen E, Gustafsson S, Ingelsson E. Associations of fitness, physical activity, strength, and genetic risk with cardiovascular disease longitudinal analyses in the UK biobank study. Circulation. 2018;137:2583-91. https://doi. org/10.1161/CIRCULATIONAHA.117.032432.

36. Carlsson S, Ahlbom A, Lichtenstein P, Andersson T. Shared genetic influence of BMI, physical activity and type 2 diabetes: a twin study. Diabetologia. 2013;56:1031-5. https://doi.org/10.1007/s00125-013-2859-3

37. Hjerkind KV, Stenehjem JS, Nilsen TIL. Adiposity, physical activity and risk of diabetes mellitus: prospective data from the population-based HUNT study. Norway Bmj Open. 2017;7:e013142. https://doi.org/10.1136/bmjopen-2016013142

38. Jefferis BJ, Lennon L, Whincup PH, Wannamethee SG. Longitudinal associations between changes in physical activity and onset of type 2 diabetes in older British men: the influence of adiposity. Diabetes Care. 2012;35:1876-83.

39. Koloverou E, Panagiotakos DB, Pitsavos C, Chrysohoou C, Georgousopoulou EN, Tousoulis D, Stefanadis C. The long term effect of dietary habits and physical activity on type 2 diabetes incidence: 10-year follow up of the ATTICA study (2002-2012): diet, physical activity and diabetes. Hellenic J Atherosclerosis. 2018;9:5-16

40. Elwood P, Galante J, Pickering J, Palmer S, Bayer A, Ben-Shlomo Y, et al. Healthy lifestyles reduce the incidence of chronic diseases and dementia: evidence from the Caerphilly cohort study. PLoS One. 2013;8:e81877. https://doi.org/10.1371/journal.pone.0081877.

41. Grøntved A, Pan A, Mekary RA, Stampfer M, Willett WC, Manson JE, Hu FB. Muscle-strengthening and conditioning activities and risk of type 2 diabetes: a prospective study in two cohorts of US women. PLOS Med. 2014;11:e1001587. https://doi.org/10.1371/journal.pmed.1001587.

42. Ekelund U, Palla L, Brage S, Franks PW, Peters T, Balkau B, et al. Physical activity reduces the risk of incident type 2 diabetes in general and in abdominally lean and obese men and women: the EPIC-InterAct study. Diabetologia. 2012;55:1944-52. https://doi.org/10.1007/s00125-012-2532-2 .

43. Mehlig K, Skoog I, Waern M, Miao Jonasson J, Lapidus L, Björkelund C, et al. Physical activity, weight status, diabetes and dementia: a 34-year follow-up of the population study of women in Gothenburg. Neuroepidemiology. 2014;42:252-9. https://doi.org/10.1159/000362201

44. Shi L, Shu X-O, Li H, Cai H, Liu Q, Zheng W, et al. Physical activity, smoking, and alcohol consumption in association with incidence of type 2 diabetes among middle-aged and elderly Chinese men. PLoS One. 2013;8:e77919. https://doi.org/10.1371/journal.pone.0077919.

45. Lu Y, Lu M, Dai H, Yang P, Smith-Gagen J, Miao R, et al. Lifestyle and risk of hypertension: follow-up of a young pre-hypertensive cohort. Int J Med Sci. 2015;12:605-12. https://doi.org/10.7150/ijms.12446

46. Pavey TG, Peeters G, Bauman AE, Brown WJ. Does vigorous physical activity provide additional benefits beyond those of moderate? Med Sci Sports Exerc. 2013;45:1948-55. https://doi.org/10.1249/MSS.0b013e3182940b91

47. Stenehjem JS, Hjerkind KV, Nilsen TIL. Adiposity, physical activity, and risk of hypertension: prospective data from the population-based HUNT study, Norway. J Human Hypertens. 2018;32:278-86.

48. Cohen L, Curhan GC, Forman JP. Influence of age on the association between lifestyle factors and risk of hypertension. J Am Soc Hypertens. 2012;6:284-90. https://doi.org/10.1016/j.jash.2012.06.002

49. Medina C, Janssen I, Barquera S, Bautista-Arredondo S, Gonzalez ME, Gonzalez C. Occupational and leisure time physical inactivity and the risk of type II diabetes and hypertension among Mexican adults: a prospective cohort study. Sci Rep. 2018. https://doi.org/10.1038/s41598-018-23553-6

50. Williams PT, Thompson PD. Walking versus running for hypertension, cholesterol, and diabetes mellitus risk reduction. Arterioscler Thromb Vasc Biol. 2013;33:1085-91. https://doi.org/10.1161/ATVBAHA.112.300878. 
51. Aune D, Norat T, Leitzmann M, Tonstad S, Vatten LJ. Physical activity and the risk of type 2 diabetes: a systematic review and dose-response meta-analysis. Eur J Epidemiol. 2015;30:529-42. https://doi.org/10.1007/s10654-015-0056-z .

52. Sattelmair J, Pertman J, Ding EL, Kohl HW, Haskell W, Lee I-M. Dose response between physical activity and risk of coronary heart disease: a meta-analysis. Circulation. 2011;124:789-95. https://doi.org/10.1161/ CIRCULATIONAHA.110.010710

53. Chin S-H, Kahathuduwa CN, Binks M. Physical activity and obesity: what we know and what we need to know. Obes Rev. 2016;17:1226-44. https://doi. org/10.1111/obr.12460.

54. Shaw K, Gennat H, O'Rourke P, Del Mar C. Exercise for overweight or obesity. Cochrane Database Syst Rev. 2006:CD003817. https://doi.org/10. 1002/14651858.CD003817.pub3 .

55. Tam G, Yeung MPS. A systematic review of the long-term effectiveness of work-based lifestyle interventions to tackle overweight and obesity. Prev Med. 2018;107:54-60. https://doi.org/10.1016/j.ypmed.2017.11.011

56. Rodrigues AL, Ball J, Ski C, Stewart S, Carrington MJ. A systematic review and meta-analysis of primary prevention programmes to improve cardiometabolic risk in non-urban communities. Prev Med. 2016;87:22-34. https:// doi.org/10.1016/j.ypmed.2016.02.011

57. Wilks DC, Besson H, Lindroos AK, Ekelund U. Objectively measured physical activity and obesity prevention in children, adolescents and adults: a systematic review of prospective studies. Obes Rev. 2011;12:e119-29. https://doi.org/10.1111/j.1467-789X.2010.00775.X

58. Liu X, Zhang D, Liu Y, Sun X, Han C, Wang B, et al. Dose-response association between physical activity and incident hypertension: a systematic review and meta-analysis of cohort studies. Hypertension. 2017; 69:813-20. https://doi.org/10.1161/HYPERTENSIONAHA.116.08994

59. Huai P, Xun H, Reilly KH, Wang Y, Ma W, Xi B. Physical activity and risk of hypertension: a meta-analysis of prospective cohort studies. Hypertension. 2013;62:1021-6. https://doi.org/10.1161/HYPERTENSIONAHA.113.01965 .

60. Lee I-M, Shiroma EJ, Evenson KR, Kamada M, LaCroix AZ, Buring JE. Accelerometer-measured physical activity and sedentary behavior in relation to all-cause mortality: the Women's health study. Circulation. 2018;137:2035. https://doi.org/10.1161/CIRCULATIONAHA.117.031300 .

61. LaCroix AZ, Bellettiere J, Rillamas-Sun E, Di C, Evenson KR, Lewis CE, et al. Association of Light Physical Activity Measured by Accelerometry and incidence of coronary heart disease and cardiovascular disease in older women. JAMA Netw Open. 2019;2:e190419. https://doi.org/10.1001/ jamanetworkopen.2019.0419.

62. Colberg SR, Sigal RJ, Fernhall B, Regensteiner JG, Blissmer BJ, Rubin RR, et al: Exercise and type 2 diabetes: the American College of Sports Medicine and the American Diabetes Association: joint position statement. Diabetes Care. 2010;33:e147-67. https://doi.org/10.2337/dc10-9990

63. Jakicic JM, Rogers RJ, Davis KK, Collins KA. Role of physical activity and exercise in treating patients with overweight and obesity. Clin Chem. 2018; 64:99-107. https://doi.org/10.1373/clinchem.2017.272443.

64. Winzer EB, Woitek F, Linke A. Physical activity in the prevention and treatment of coronary artery disease. J Am Heart Assoc. 2018. https://doi. org/10.1161/JAHA.117.007725

65. Mnich C. Is there Europeanization of physical activity promotion? - a neofunctional approach. Health Policy. 2019;123:317-26. https://doi.org/10. 1016/j.healthpol.2019.01.004

\section{Publisher's Note}

Springer Nature remains neutral with regard to jurisdictional claims in published maps and institutional affiliations.

Ready to submit your research? Choose BMC and benefit from:
- fast, convenient online submission
- thorough peer review by experienced researchers in your field
- rapid publication on acceptance
- support for research data, including large and complex data types
- gold Open Access which fosters wider collaboration and increased citations
- maximum visibility for your research: over 100M website views per year
At BMC, research is always in progress.
Learn more biomedcentral.com/submissions

